\title{
1 Nano-scale Architecture of Blood-Brain Barrier Tight-Junctions
}

2 Esther Sasson ${ }^{1}$, Shira Anzi ${ }^{1}$, Batia Bell ${ }^{1}$, Oren Yakovian $^{2}$, Meshi Zorsky ${ }^{3}$, Urban Deutsch ${ }^{4}$, 3 Britta Engelhardt ${ }^{4}$, Eilon Sherman ${ }^{2}$, Gad D. Vatine $^{3}$, Ron Dzikowski ${ }^{5}$, Ayal Ben-Zvi ${ }^{*}$.

1. Department of Developmental Biology and Cancer Research, The Institute for Medical

6 Research Israel-Canada, Faculty of Medicine, Hebrew University of Jerusalem, Jerusalem 7 91120, Israel

8

2. Racah Institute of Physics, The Hebrew University, Jerusalem 9190401, Israel

3. The Department of Physiology and Cell Biology, Faculty of Health Sciences, The Regenerative Medicine and Stem Cell (RMSC) research Center and the Zlotowski Center for Neuroscience, Ben-Gurion University of the Negev, Beer Sheva 84105, Israel

4. Theodor Kocher Institute, University of Bern, Bern, Switzerland. Infectious and Tropical Diseases, IMRIC, Faculty of Medicine, Hebrew University of

19 Jerusalem, Jerusalem 91120, Israel.

\section{* Address correspondence to:}

23 Ayal Ben-Zvi, PhD

Department of Developmental Biology and Cancer Research,

25 The Institute for Medical Research Israel-Canada,

26 Faculty of Medicine,

27 Hebrew University of Jerusalem,

28 Jerusalem 91120, Israel

Tel: $+972-2-6787624$ 


\section{Abstract}

Tight junctions (TJs) between blood-brain barrier (BBB) endothelial cells construct a robust physical barrier, whose damage underlies BBB dysfunctions related to several neurodegenerative diseases. What makes these highly specialized BBB-TJs extremely restrictive remains unknown. Here, we use super-resolution microscopy (dSTORM) to uncover new structural and functional properties of BBB TJs. Focusing on three major components, Nano-scale resolution revealed sparse (occludin) vs. clustered (ZO1/claudin-5) molecular architecture. Developmentally, permeable TJs become first restrictive to large molecules, and only later to small molecules, with claudin-5 proteins arrangement compacting during this maturation process. Mechanistically, we reveal that $\mathrm{ZO1}$ clustering is independent of claudin-5 in-vivo. In contrast to accepted knowledge, we found that in the developmental context, total levels of claudin-5 inversely correlate with $\mathbf{T J}$ functionality. Our super-resolution studies provide a unique perspective of BBB TJs and open new directions for understanding TJ functionality in biological barriers, ultimately enabling restoration in disease or modulation for drug delivery.

\section{Introduction}

The blood-brain barrier (BBB) was identified when dye/tracer injected into the blood circulation reached the majority of body tissues but failed to penetrate the brain (Hagan \& Ben-Zvi, 2015). Endothelial cells (ECs) were identified as the core component of the mammalian BBB when electron microscopy (EM) allowed imaging of fine ultra-structural cell-biology components of BBB cells (Brightman \& Reese, 1969; Reese \& Karnovsky, 1967). Horseradish-peroxidase (HRP) used as an EM compatible tracer revealed that BBB 
rates of transcytosis (vesicular transport), both mediating intracellular permeability in peripheral ECs. A central discovery of these studies was that tight junctions (TJs) between neighboring ECs are responsible for intercellular restrictive barrier properties (Brightman \& Reese, 1969; Reese \& Karnovsky, 1967). Ever since, BBB TJs became a major focus of the $\mathrm{BBB}$ research field; TJs modulation is explored as means to enhance brain drug delivery, and TJs damage is investigated to better understand underlying BBB dysfunctions implicated in diseases (neurodegenerative, neuro-inflammatory, trauma etc. (Bauer, Krizbai, Bauer, \& Traweger, 2014; Greene et al., 2018; Hagan \& Ben-Zvi, 2015; Kealy, Greene, \& Campbell, 2018; Knowland et al., 2014; Liebner et al., 2018; Sweeney, Sagare, \& Zlokovic, 2018; Zhao, Nelson, Betsholtz, \& Zlokovic, 2015; Zlokovic, 2008)).

Several gene families encoding integral membrane proteins (e.g occludin, junctional adhesion molecules (JAMs), claudins, and tricellulins (LSR/Marveld)) and adaptors that link TJs to the cytoskeleton (such as zonula occludens (ZO)), participate in constructing BBB TJs (Bauer et al., 2014; Furuse, Fujita, Hiiragi, Fujimoto, \& Tsukita, 1998; Furuse et al., 1993; Haseloff, Dithmer, Winkler, Wolburg, \& Blasig, 2015; Knowland et al., 2014; Langen, Ayloo, \& Gu, 2019; Martin-Padura et al., 1998; Morita, Sasaki, Furuse, \& Tsukita, 1999; Nitta et al., 2003; Sohet et al., 2015). Immunofluorescence and imaging with conventional light microscopy provided insights into the molecular components of TJs. Nevertheless, these approaches do not enable proper resolution for nano-scale imaging of TJ architecture. Electron microscopy on the other hand, provides superb resolution to image cellular structures but is much less effective in simultaneously localizing multiple proteins and tracer molecules, limiting our ability to study the molecular architecture of TJs. In order to bridge the gap between these two imaging methodologies and overcome their limitations, we developed an approach to image the $\mathrm{BBB}$ with direct stochastic optical reconstruction microscopy (dSTORM (van de Linde et al., 2011)) and study TJs at the nano-scale level. 


\section{Results}

\section{Super-resolution microscopy of endothelial tight junctions}

We hypothesized that effective imaging of multiple TJ proteins organized in a very tight spatial localization could be achieved with dSTORM. To evaluate this approach we used bEND.3 cells (a mouse brain-derived endothelioma cell-line), cultured in vitro to form a confluent monolayer. These cells were shown to form TJs in a gradual process that includes translocation of claudin-5 from cytoplasmic and general membrane localizations to the cell boundaries in contact between adjacent cells (Koto et al., 2007). We confirmed these observations with immunofluorescence labeling claudin-5 and ZO1 in several time points after the cultures reached confluence state (Fig. 1a). Previous studies also demonstrated that during this process, claudin-5 expression is increased reaching maximal levels by three days post-confluence. The monolayer trans-endothelial electrical resistance (TEER), a proxy for TJ function reflecting intercellular restrictive properties, is elevated reaching maximal levels by seven days post-confluence (Koto et al., 2007).

We therefore further investigated two states: post-confluence (3-7 days post-confluence) and super-confluence (more than 11 days post-confluence). Using antibodies against claudin5 and ZO1 for immunofluorescence and ASTORM imaging, we demonstrated a new layer of complexity in TJ organization (Fig. 1b-e). Most TJ studies use the term 'strands' to describe the organization of $\mathrm{TJ}$ proteins in a continuous line around the boundaries of the cells (as seen in Fig. 1a). Super resolution imaging allowed us to demonstrate that both claudin-5 (green) and ZO1 (red) are not organized in continuous lines but rather in disrupted lines with discrete clusters, forming bead-like structures (Fig. 1b). A pronounced change was found in the organization of the two TJ proteins at the cell-cell contacts: at post-confluence, signals along the membrane were more diffused, forming elongated clusters and $\mathrm{ZO} 1$ signals intermingled with claudin-5 signals (Fig. 1b, arrows). In contrast, at super-confluence, claudin-5 signals 
106 along the membrane became concentrated in more discrete and shorter foci, flanking ZO1

107 signals (Fig. 1b, arrowheads).

108 Target proteins labeled with antibodies imaged with dSTORM produced resolved signals

109 representing an amplification of actual target numbers (see methods for details). Resolution of

110 approximately $20 \mathrm{~nm}$ allowed us to separate signals and to use these as proxies for the

111 abundance of target molecules, which could be used to compare different states. To quantify

112 the differences in TJ architecture during this in vitro process, we analyzed the images using a

113 custom clustering Matlab code to measure cluster area and number of signals per cluster.

114 These were used to calculate the signal densities for each cluster (see clustering simulation

115 Fig. 1c and methods for details). We could detect a clear shift towards smaller clusters with

116 more claudin-5 signals per cluster at super-confluence (Fig. 1d). Clusters with higher numbers

117 of signals were more abundant at this late state, especially in clusters with area smaller than

$1180.3 \mu^{2}$. Average signal density (per cluster) was also higher at the super-confluence state

119 ( 4-fold, Fig. 1e, $\mathrm{P}<0.0001)$. 
Figure 1

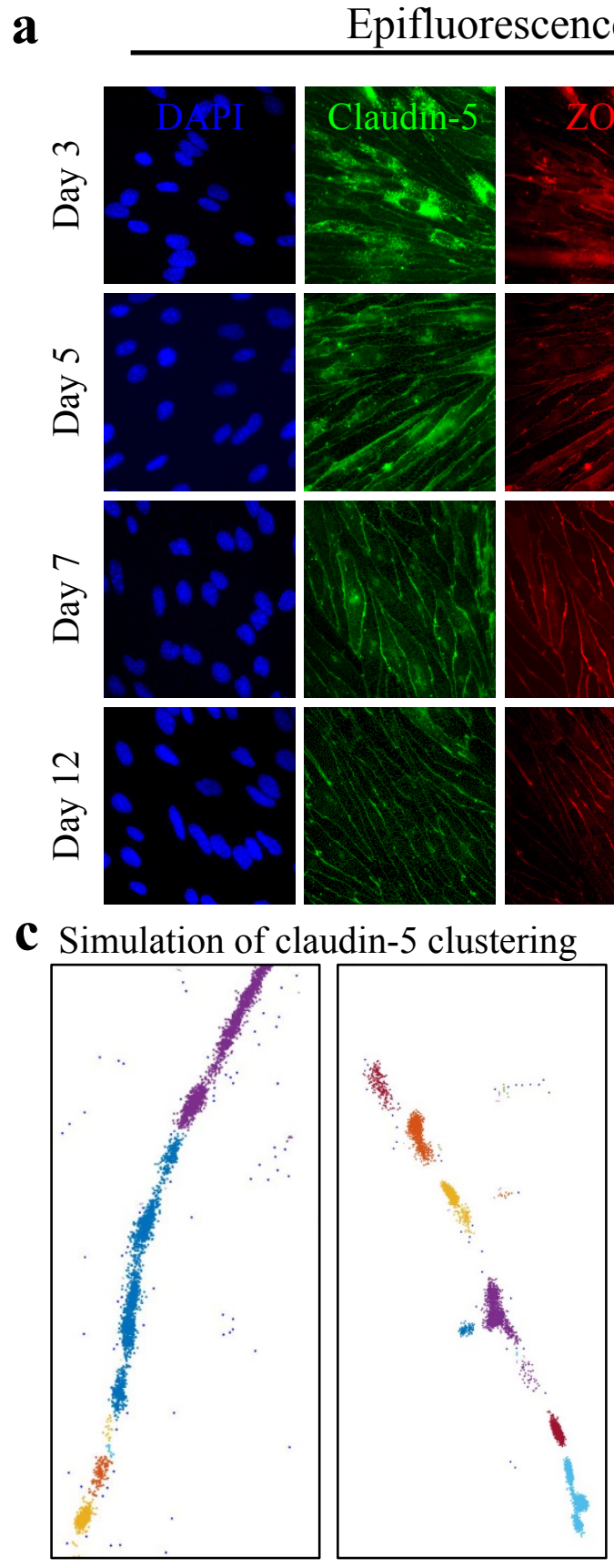

Post-confluence Super-confluence

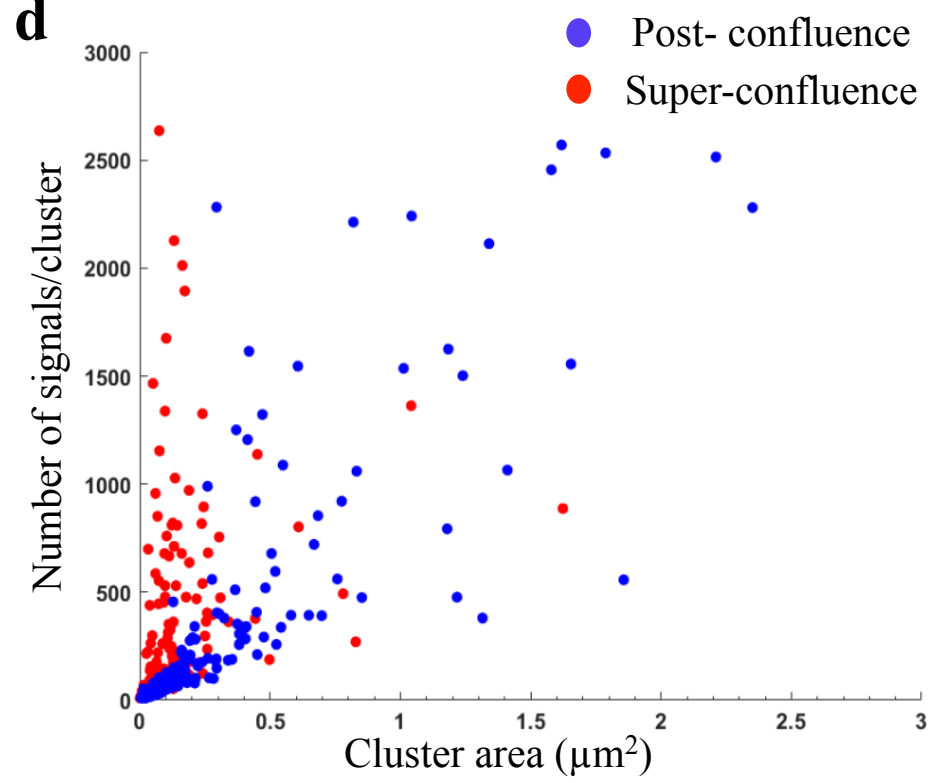

d

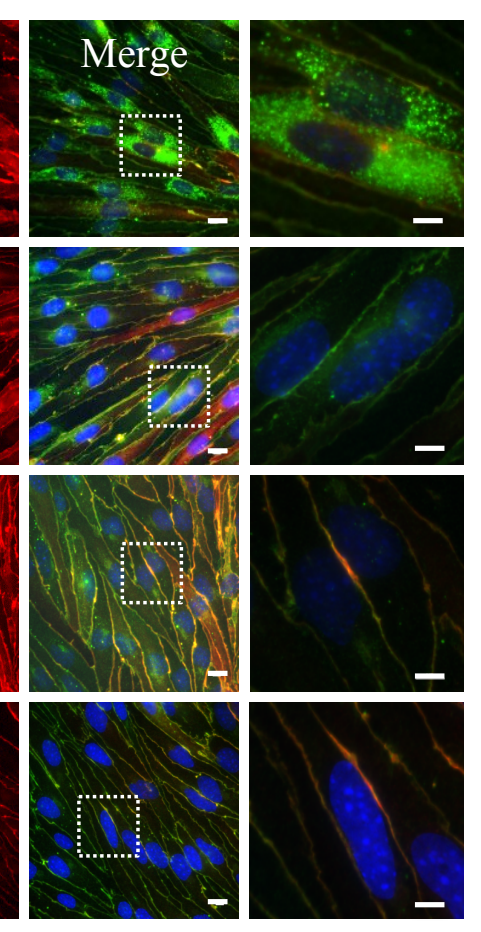

b

STORM microscopy
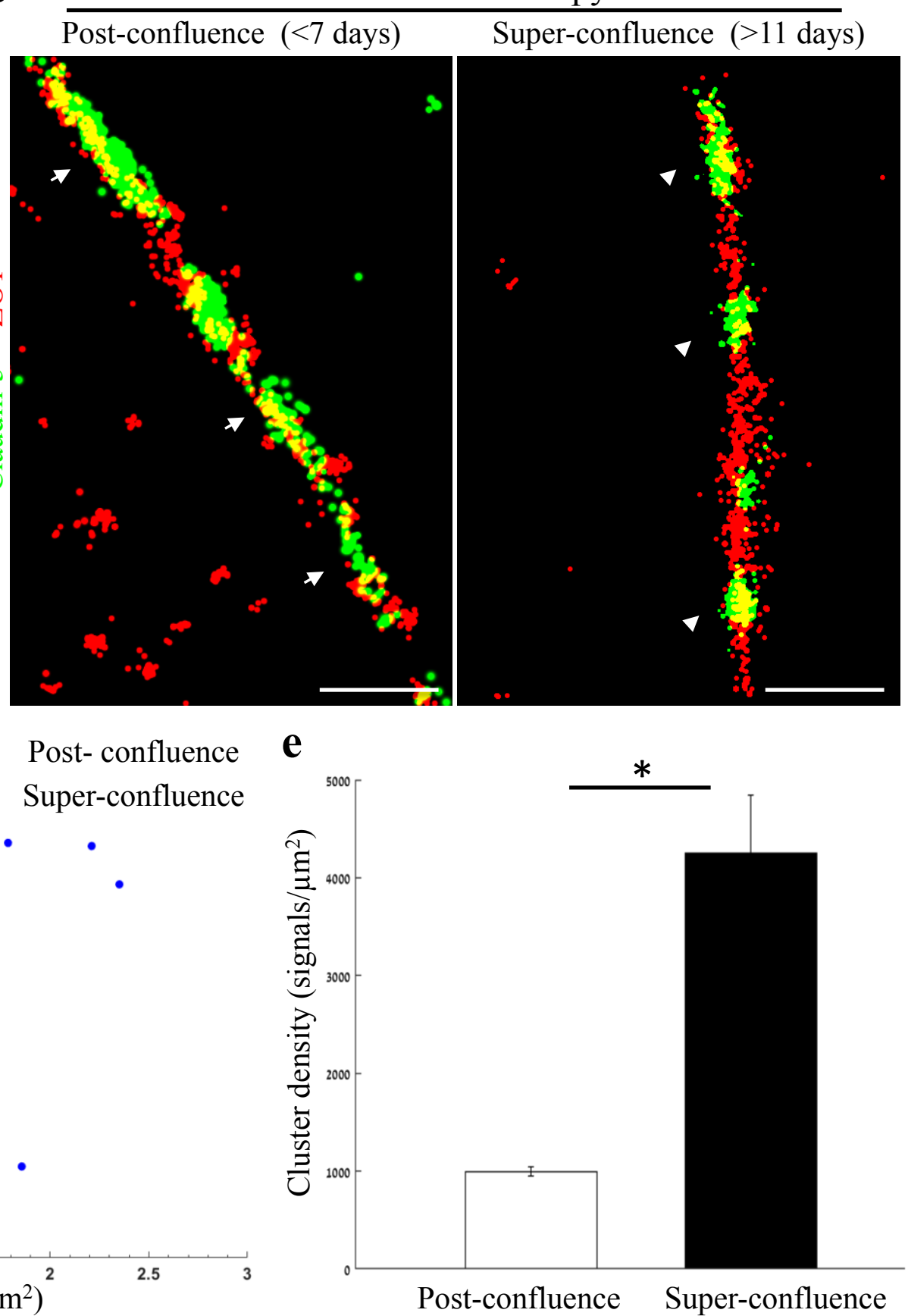
121 Fig. 1: Super-resolution microscopy of endothelial tight junctions. In vitro process of TJ

122 maturation is accompanied by TJ architectural changes characterized by the formation of

123 smaller, denser and more discrete clusters of TJ proteins. a, Epi-fluorescent imaging of

124 claudin-5 (green) and ZO1 (red) immunostaining of bEND.3 cells in indicated time points

125 after the cultures reached confluence state. Note translocation of claudin-5 from cytoplasmic

126 localizations into continuous lines around the boundaries of the cells (known as 'strands'),

127 along the in vitro maturation process. Scale bars, $10 \mu \mathrm{m}$ and $5 \mu \mathrm{m}$ in insets. b, dSTORM

128 imaging (Gaussian visualization) of claudin-5 (green) and ZO1 (red) immunostaining of

129 bEND.3 cells confluent monolayers. TJ proteins form bead-like structures, especially in the

130 super-confluence state. Claudin-5 signals are more concentrated in discrete and shorter foci

131 (flanking ZO1 signals, arrowheads, right) than in the post-confluence state (arrows, left).

132 During maturation TJ proteins translocate from different cellular locations (left) almost

133 exclusively into the lateral cell membranes (right). Scale bar, $1 \mu \mathrm{m}$. c, Examples of dSTORM

134 imaging simulation of claudin-5 in bEND. 3 cells used for quantifications of clustering

135 properties (produced by a custom clustering Matlab code, see methods for details). Signals

136 were defined to be clustered if their 2D location was smaller than $70 \mathrm{~nm}$ threshold distance.

137 Cluster pattern visualization showing all points that belong to the same cluster with the same

138 identifying color. d, Quantifications of claudin-5 clustering properties showed a shift towards

139 smaller clusters with more claudin-5 signals per cluster at the super-confluence state. Clusters

140 with higher numbers of signals were more abundant at this late state, especially in clusters

141 with area smaller than $0.3 \mu \mathrm{m}^{2}$. e, Average claudin-5 cluster density was $\sim 4$-fold higher at the

142 super-confluence state than in the post-confluence state ( $n=183$ clusters (post-confluence) and

143281 clusters (super-confluence) in 5 independent experiments). Data are mean \pm s.e.m. $* \mathrm{P}<$

1440.05 (Two tailed Mann-Whitney U-test). 
We further investigated nano-architectural changes in the in vitro context of claudin-5 expression and tight junction function. In line with previous publications (Koto et al., 2007), we could confirm that total claudin-5 protein levels in bEND. 3 cells rise with time in culture

148 (Fig. S1a). Nevertheless, we found that in general, bEND.3 monolayer trans-endothelial electrical resistance (TEER) is relatively low ( 40-100 $\Omega$ xcm2). Therefore, we turned to an 150 alternative in vitro system that presents substantially superior barrier features with 151 pronounced TJ function and TEER levels closer to those estimated for the in vivo levels: 152 human iPSC differentiation into brain microvascular endothelial-like cells (iBMECs) 153 (Lippmann, Al-Ahmad, Azarin, Palecek, \& Shusta, 2014; Lippmann et al., 2012; Vatine et al., 154 2017; Vatine et al., 2019). In our culturing conditions TEER levels started at 500-1000 $155 \Omega x c m 2$ already a day after seeding. We monitored TEER and upon a noticeable elevation of 156 approximately an additional $\sim 1000 \Omega x \mathrm{~cm} 2$ (2-3 days in culture), we measured claudin-5 157 protein levels with western blot, permeability in transwells, and in parallel imaged cultures with STORM (Fig 2). We noticed that TJ function was improving, with TEER elevation (Fig. 2a, S1b) and flux decrease (Fig. 2b), but could not detect noticeable changes in claudin-5

160 protein levels. STORM imaging revealed that only clustered organization of claudin-5 could 161 be found in these cells (Fig. 2c), and that pronounced change in nano-scale organization of 162 claudin-5 clusters could be observed; clusters were smaller in area and denser (Fig. 2d-f) 163 along with improvement in TJ function. The majority of cluster areas were smaller than 0.1 $164 \mu \mathrm{m}^{2}$, and the average signal density (per cluster) was higher with elevated TEER ( 2.3-fold, 165 Fig. 1f, $\mathrm{P}<0.01$ ). In general, clusters of claudin-5 are much denser in iBMECs compared to 166 bEND.3 cells ( 8 fold denser), which correlates with differences in TEER.

167 Altogether, we suggest that the in vitro process of TJ maturation is accompanied by TJ 168 nano-architectural changes characterized by the formation of smaller, denser and more 169 discrete clusters of TJ proteins. 
Figure 2

a

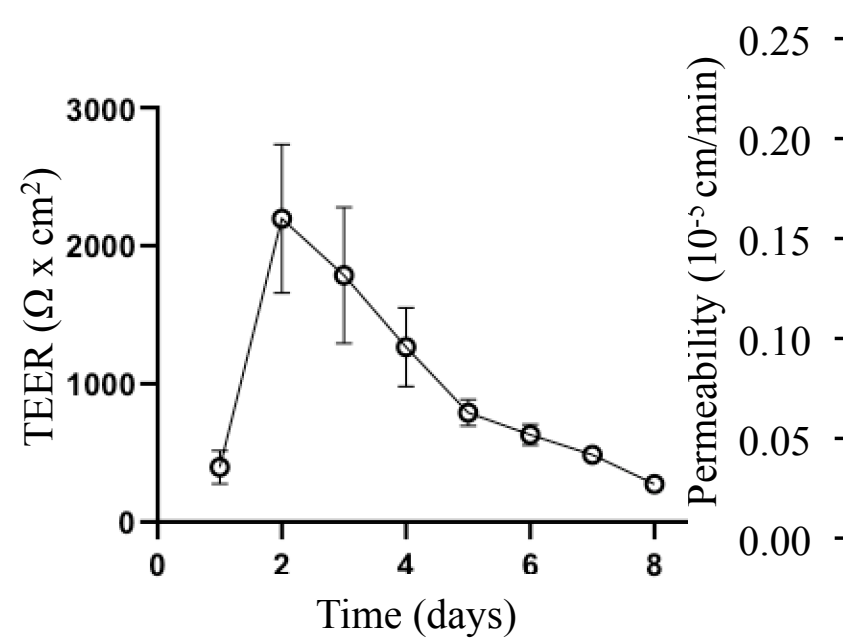

d

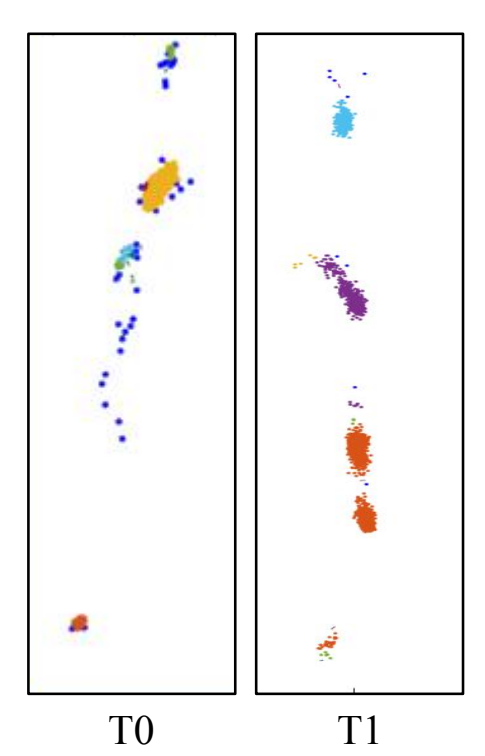

b

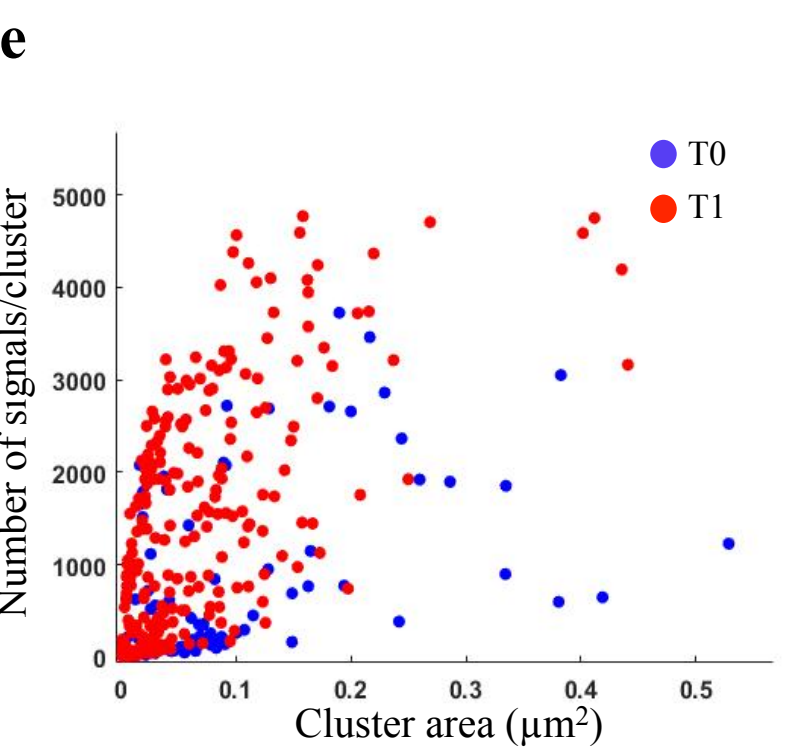

STORM microscopy

c

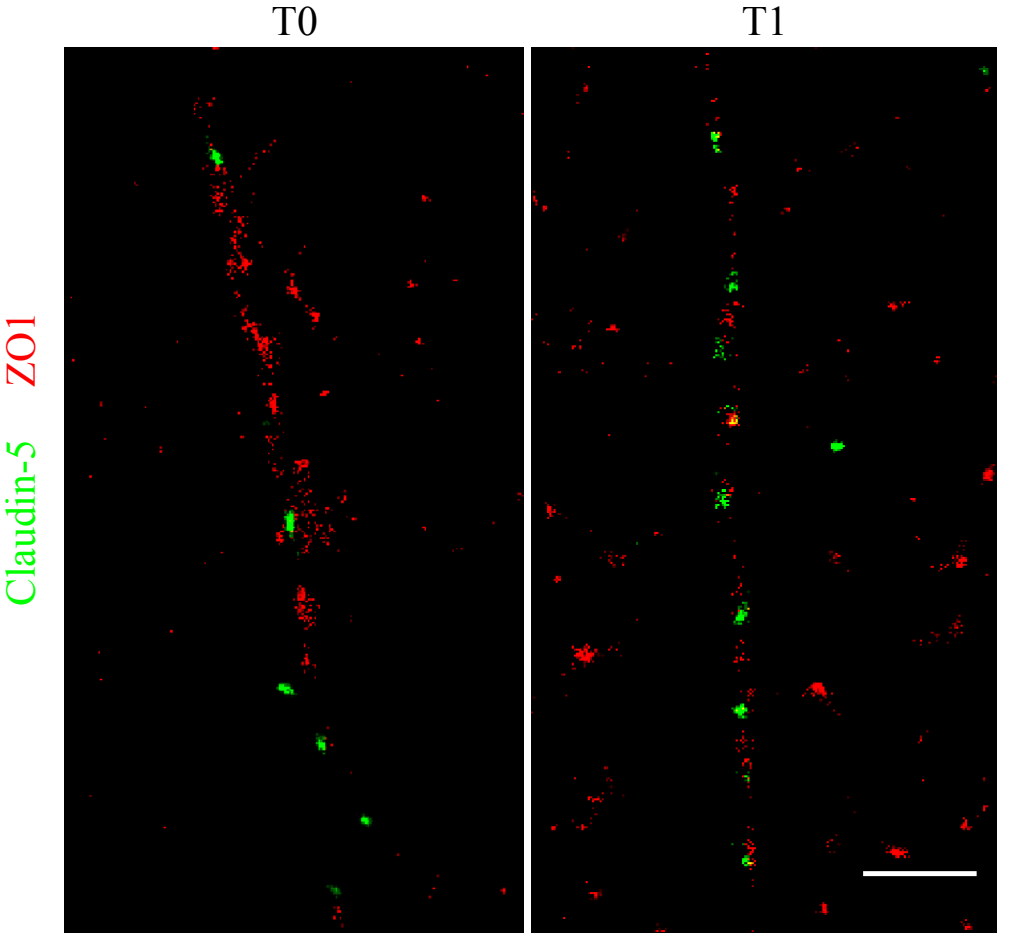

f

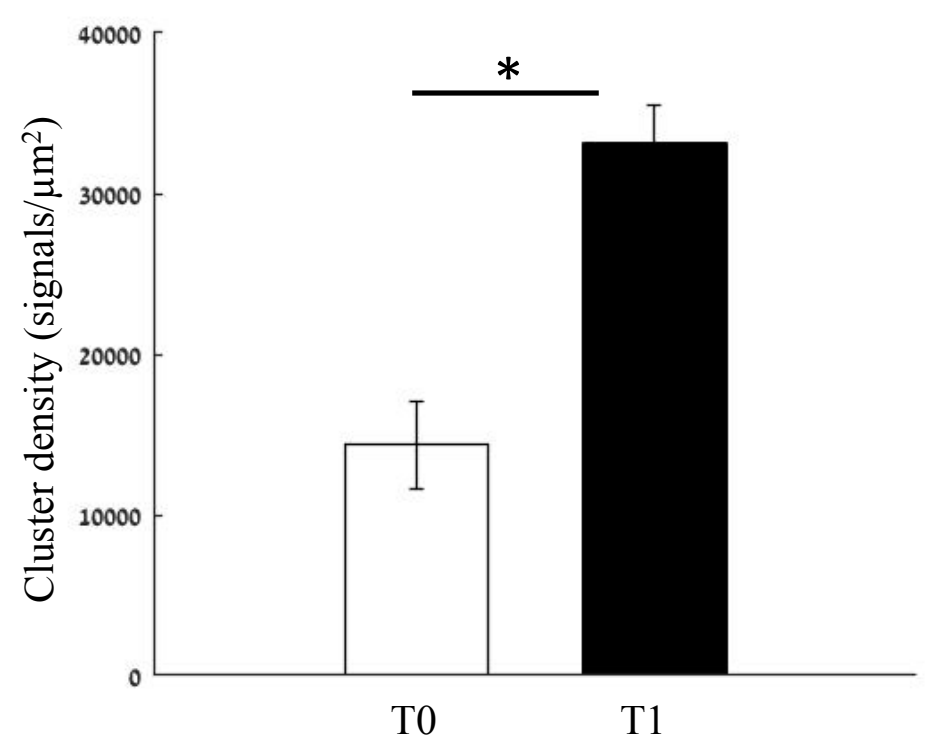


Fig. 2: Changes in nano-scale architecture correlates with tight junction function.

171 Enhanced TJ function is accompanied by formation of smaller and denser clusters of claudin-

172 5. a, Enhanced TJ function demonstrated by increase in TEER, along the first days of induced

173 human brain microvascular endothelial-like cell (iBMEC) culture ( $\mathrm{n}=4$ experiments $/ 12$

174 inserts. TEER here shows data of a representative experiment. For average change in TEER

175 across all experiments see Supplementary Figure S1b. n=4 inserts for permeability). b,

176 Enhanced TJ function demonstrated by reduced permeability to sodium fluorescein (T0

177 represents low TEER and T1 represents high TEER states, $\mathrm{n}=4$ inserts). Data are mean \pm SD.

$178 * * * \mathrm{P}<0.0003$ (two tailed pair t- test). c, dSTORM imaging (Gaussian visualization) of

179 claudin-5 (green) and ZO1 (red) immunostaining in iBMEC confluent monolayers. Claudin-5

180 signals are concentrated in discrete and short foci at both time points. Scale bar, $1 \mu \mathrm{m} . \mathbf{d}$,

181 Examples of dSTORM imaging simulation of claudin-5 in iBMECs used for quantifications

182 of clustering properties (produced by a custom clustering Matlab code, see methods for

183 details). Cluster pattern visualization showing all points that belong to the same cluster with

184 the same identifying color. e, Quantifications of claudin-5 clustering properties showed a shift

185 towards smaller clusters with more claudin-5 signals per cluster along the improvement in TJ

186 function. f, Average claudin-5 cluster density more than doubled (from 14,341 to 33,141

187 signals $\left./ \mu \mathrm{m}^{2}\right)$ with the improvement in TJ function ( $n=90$ clusters (in lower TEER) and 278

188 clusters (in higher TEER) in triplicate cultures of two independent experiments). Data are

189 mean \pm s.e.m. $*$ P $<0.01$ (two tailed Mann-Whitney U-test). 


\section{Molecular organization of mouse cortical BBB TJs}

193 To determine if the organization of $\mathrm{TJ}$ components observed in vitro occurs also in the

194 brain, we developed a technique that enabled ASTORM imaging of BBB TJ in brain tissue

195 sections (see methods for details). First we used fluorescent circulating tracers (Fig. 3b) and

196 co-staining of claudin-5 together with the endothelial-specific transcription factor ERG (Fig.

197 3c) and showed that claudin-5 dSTORM signals are exclusively localized to vascular

198 structures. We found that similar to the in vitro data, claudin-5 exhibits clustered organization

199 also in vivo, demonstrated in both cross and sagittal sections of cortical capillaries (of post-

200 natal mice, Fig. 4b). The considerably improved resolution of dSTORM imaging could be

201 appreciated when compared to epi-fluorescent images at very high magnifications of the same

202 capillary, under the same microscope settings (Fig. 4b, 5a). 
Figure 3

a

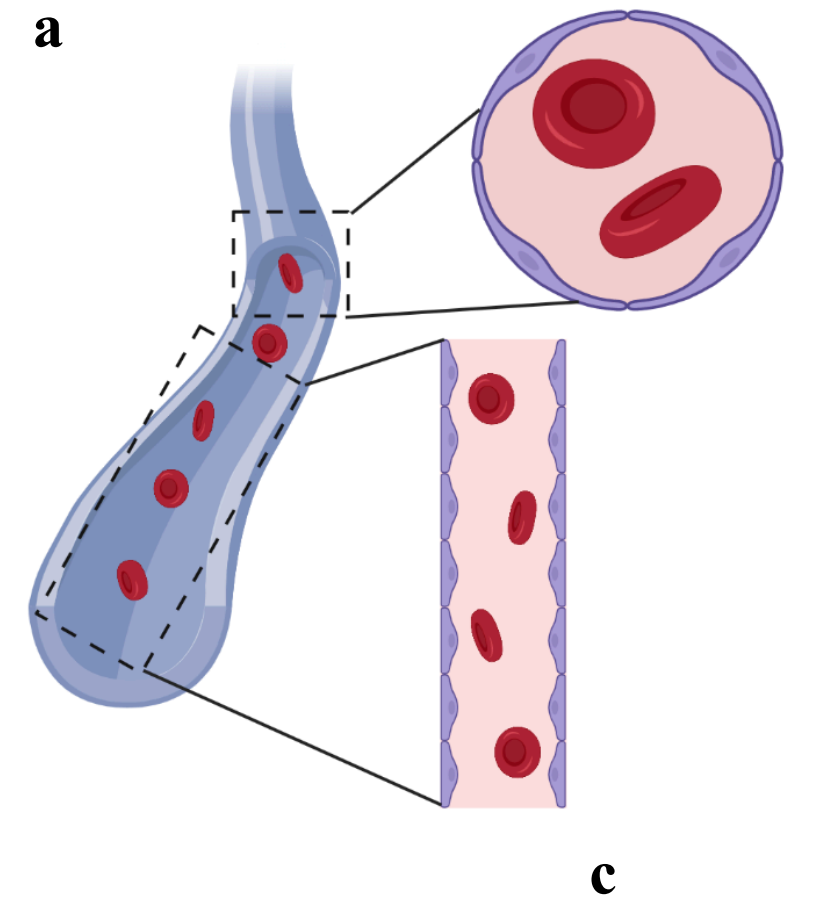

b

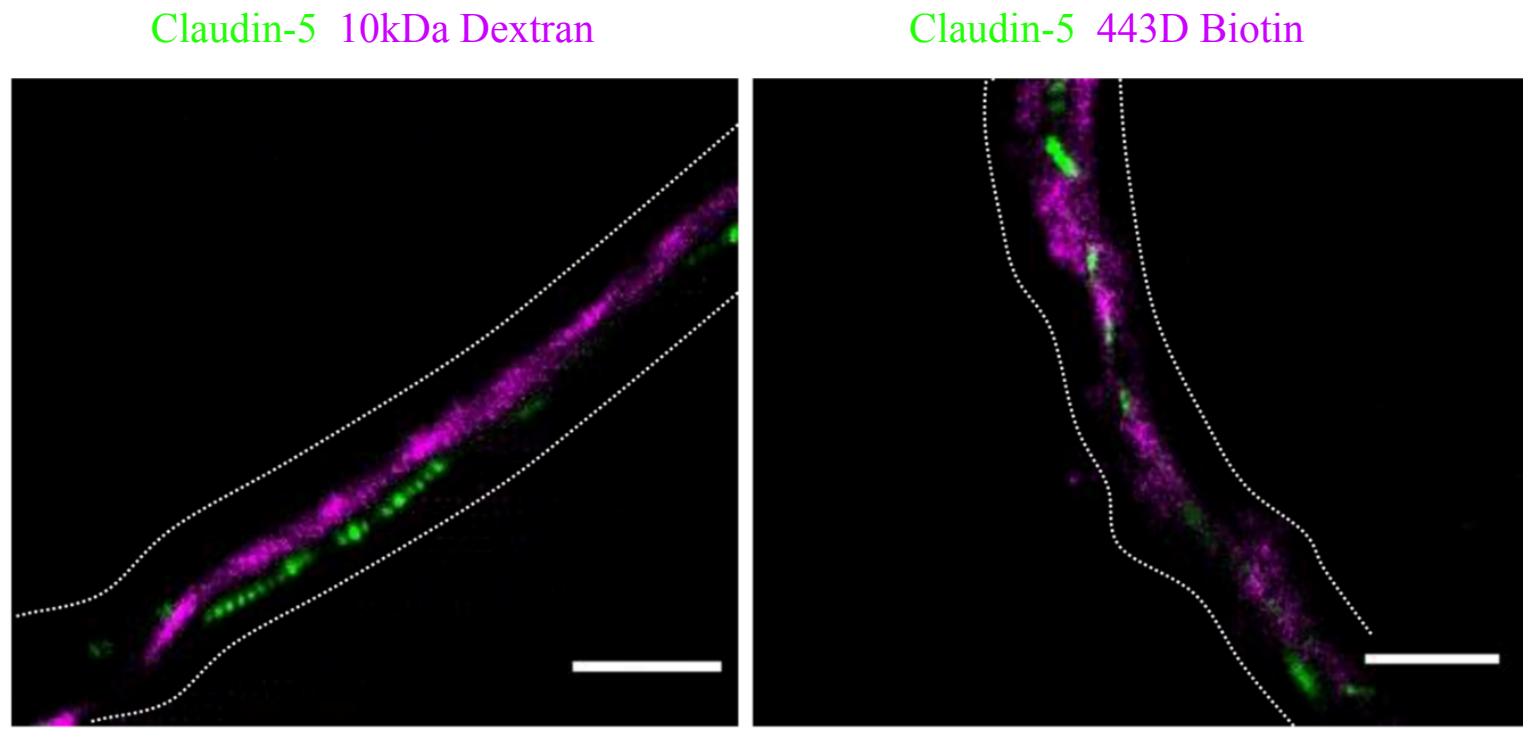

Claudin-5 ERG

Claudin-5 ERG

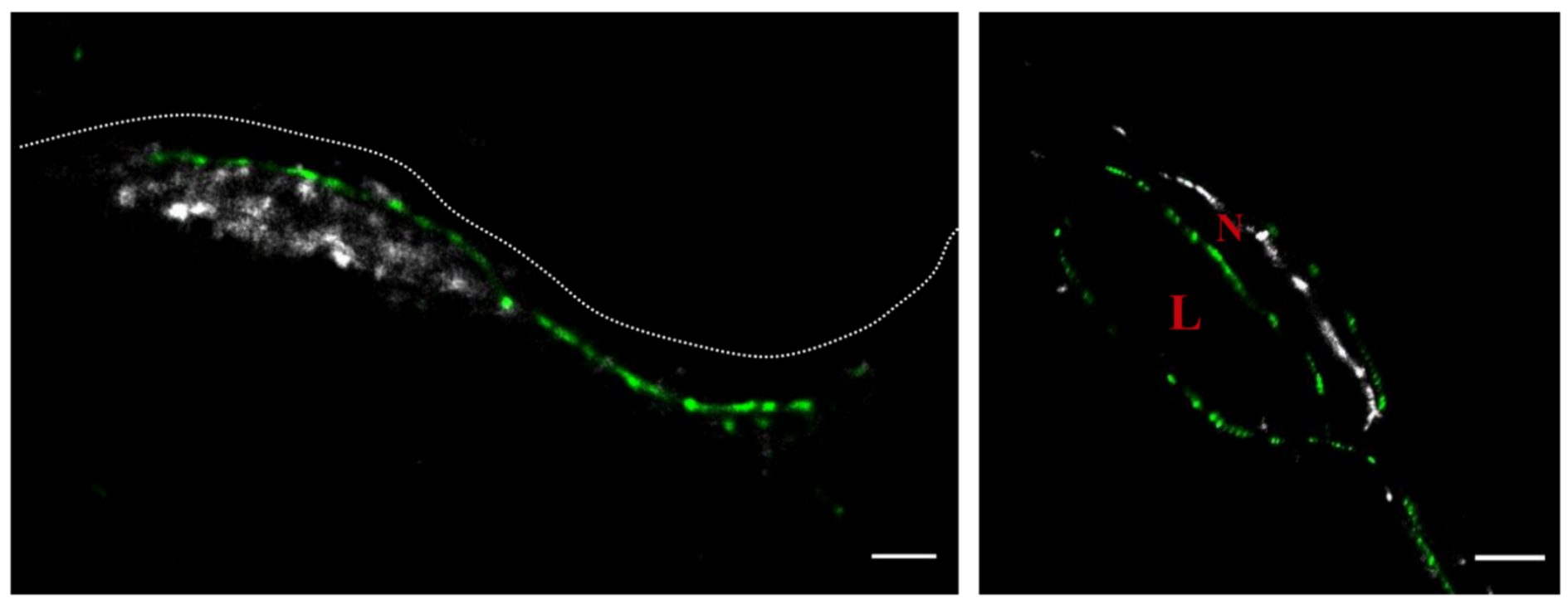


204 Fig. 3: Claudin-5 signals in dSTORM imaging are exclusively localized to vascular

205 structures. dSTORM imaging in cortical fixed tissue sections of post-natal day 9 mice. a,

206 Illustration of a vascular structure with cross versus sagittal section directions, and the

207 projected orientation of endothelial cells contact points. b, Claudin-5 staining (green) together

208 with fluorescent circulating tracers (10 kDa dextran, and 443 Dalton sulfo-NHS-biotin,

209 magenta) are used to demarcate sagittal views of elongated vessels (dashed line, Scale bars, 1

$210 \mu \mathrm{m}$ ). c, Staining for claudin-5 (green) together with the endothelial-specific transcription

211 factor ERG (gray) showing capillary cross and sagittal sections (Scale bars, $1 \mu \mathrm{m}$ ). L -

212 capillary lumen, $\mathrm{N}-$ endothelial nucleus. $n>30$ capillaries. 
Figure 4

a

Epifluorescence low magnification

b

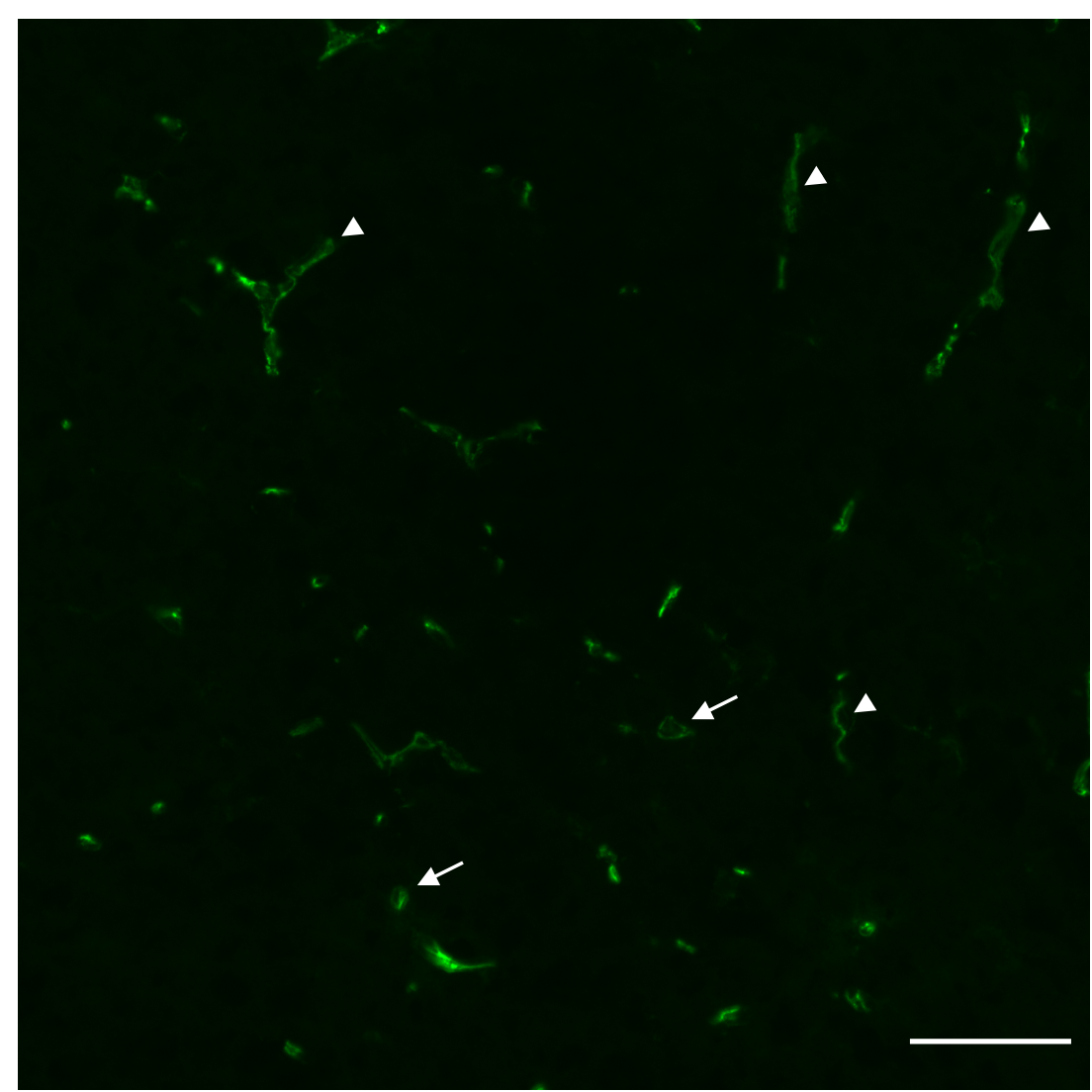

Epifluorescence

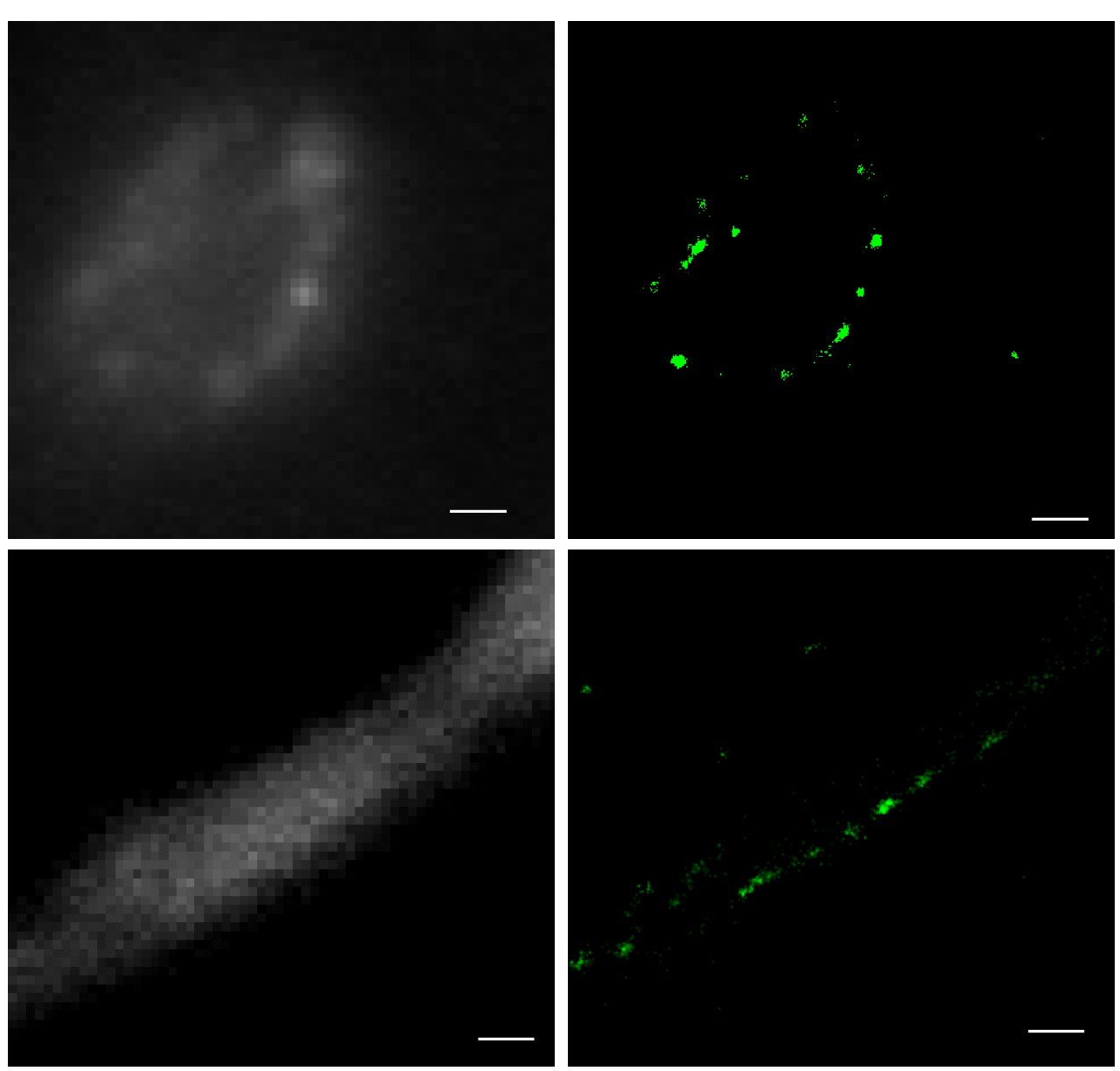


214 Fig. 4: Claudin-5 exhibits clustered organization in cortical capillaries. Imaging in

215 cortical fixed tissue sections of post-natal day 9 mice. a, Low magnification view of claudin-5

216 staining imaged by epi-fluorescent microscopy showing vascular fragments in cross-sections

217 (arrows) or in sagittal-sections (arrowheads, scale bar, $50 \mu \mathrm{m}$ ). b, Claudin-5 exhibits clustered

218 organization in vivo; dSTORM (right) compared to epi-fluorescent images (left) of claudin-5

219 immunostaining in P9 cortical capillary cross-section (upper panel) or sagittal- section (lower

220 panel) (scale bars, $1 \mu \mathrm{m}), n>30$ capillaries.

Embryonic and post-natal development of the BBB provides an opportunity to investigate in vivo TJs maturation; as cortical capillaries acquire their restrictive barrier properties along a gradual developmental process (Ben-Zvi et al., 2014; Butt, Jones, \& Abbott, 1990; Daneman,

225 Zhou, Kebede, \& Barres, 2010; Hagan \& Ben-Zvi, 2015; Langen et al., 2019; Saunders,

226 Liddelow, \& Dziegielewska, 2012; Sohet et al., 2015). From mouse embryonic day 12 (E12)

227 until E15, we and others have previously shown that the newly formed cortical capillaries 228 have not yet acquired their full restrictive barrier properties (Ben-Zvi et al., 2014; Daneman et 229 al., 2010). We therefore compared claudin-5 organization at E12 and at post-natal day 9 (P9) 230 by analyzing cellular abundance and clustering properties (Fig 5, S2). We assumed that E12 231 capillaries had a more defused claudin-5 appearance with longer clusters (Fig. 5a, arrow), but 232 further analysis revealed that these were composed of many small clusters with relatively 233 small gaps between them (example of the two types of clustering simulations, Fig. S2a).

234 Claudin-5 clustering properties analysis showed that there were about 2.6 times more discrete 235 clusters per capillary at E12 than at P9 (657 vs. 246 clusters in a set of 20 capillaries of each 236 age, Fig. 5b). Capillary diameter was also significantly larger in E12 than in P9 (Fig 5a,c; $23711.1 \pm 0.47 \mu \mathrm{m}$ and $5.9 \pm 0.39 \mu \mathrm{m}$ respectively (mean \pm s.e.m), $\mathrm{P}<0.0001$ ), which might explain 238 the difference in numbers of clusters per capillary. 
Distribution of claudin-5 clusters area in E12 capillaries was skewed towards smaller

240 clusters (with no dramatic difference between the distributions, Fig. 5b). There was no

241 dramatic difference also in the distribution of signals per cluster (Fig. 5b) or signal densities

242 between the two groups (Fig. S2b). Thus there were no obvious changes in claudin-5

243 clustering properties that correlated with changes in TJs maturation.

244 In addition to changes in capillary diameter the total number of claudin-5 signals per

245 capillary was significantly higher in E12 than at P9 $(14,341 \pm 1,257$ and 3,590 \pm 372

246 respectively (mean \pm s.e.m), $\mathrm{P}<0.0001$, Fig. 5c). Normalizing the total number of signals per

247 capillary to its diameter (displayed as 'Normalized cellular Claudin-5', Fig. 5c,d) resulted in a

248 similar significant difference (Fig. 5d). Based on this result we suggest that the total cellular

249 amount of claudin-5 is not a strong predictor of TJ functionality. Our conclusion relates to the

250 developmental and early post-natal BBB (reflected in our data), which might be distinct from

251 the adult BBB. 


\section{Figure 5}

a

Epifluorescence

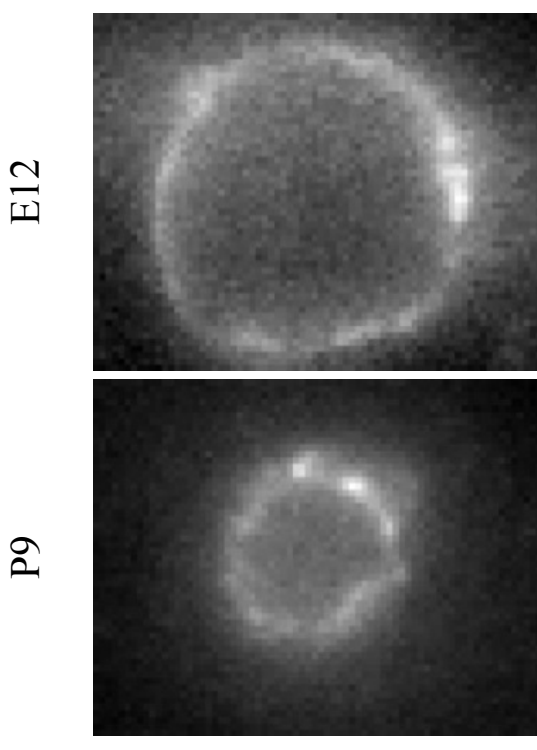

c

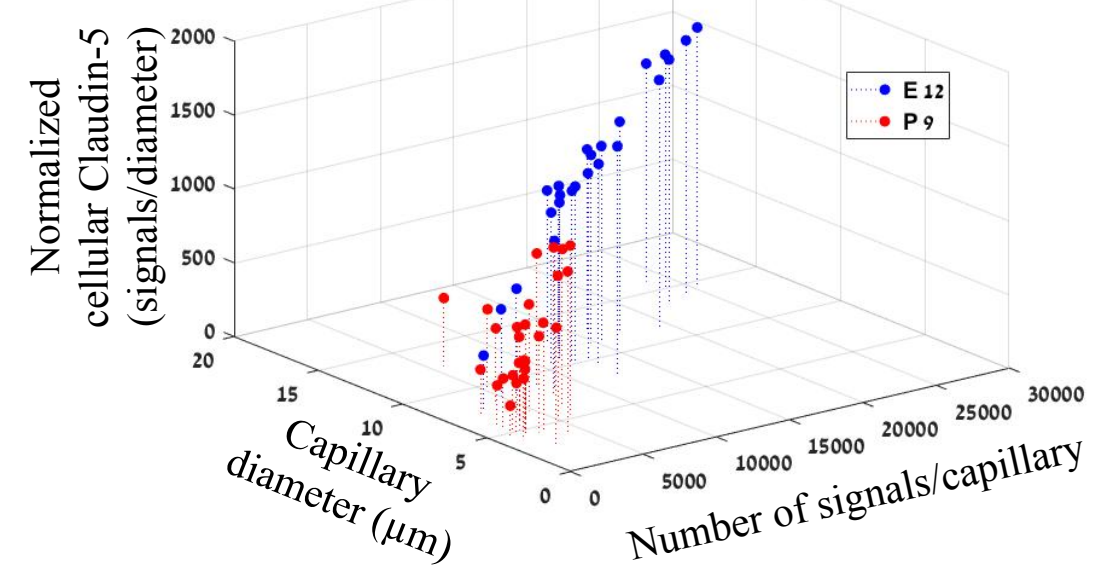

b

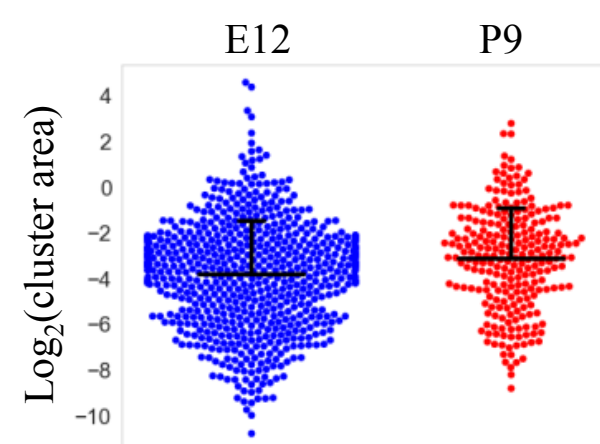

d
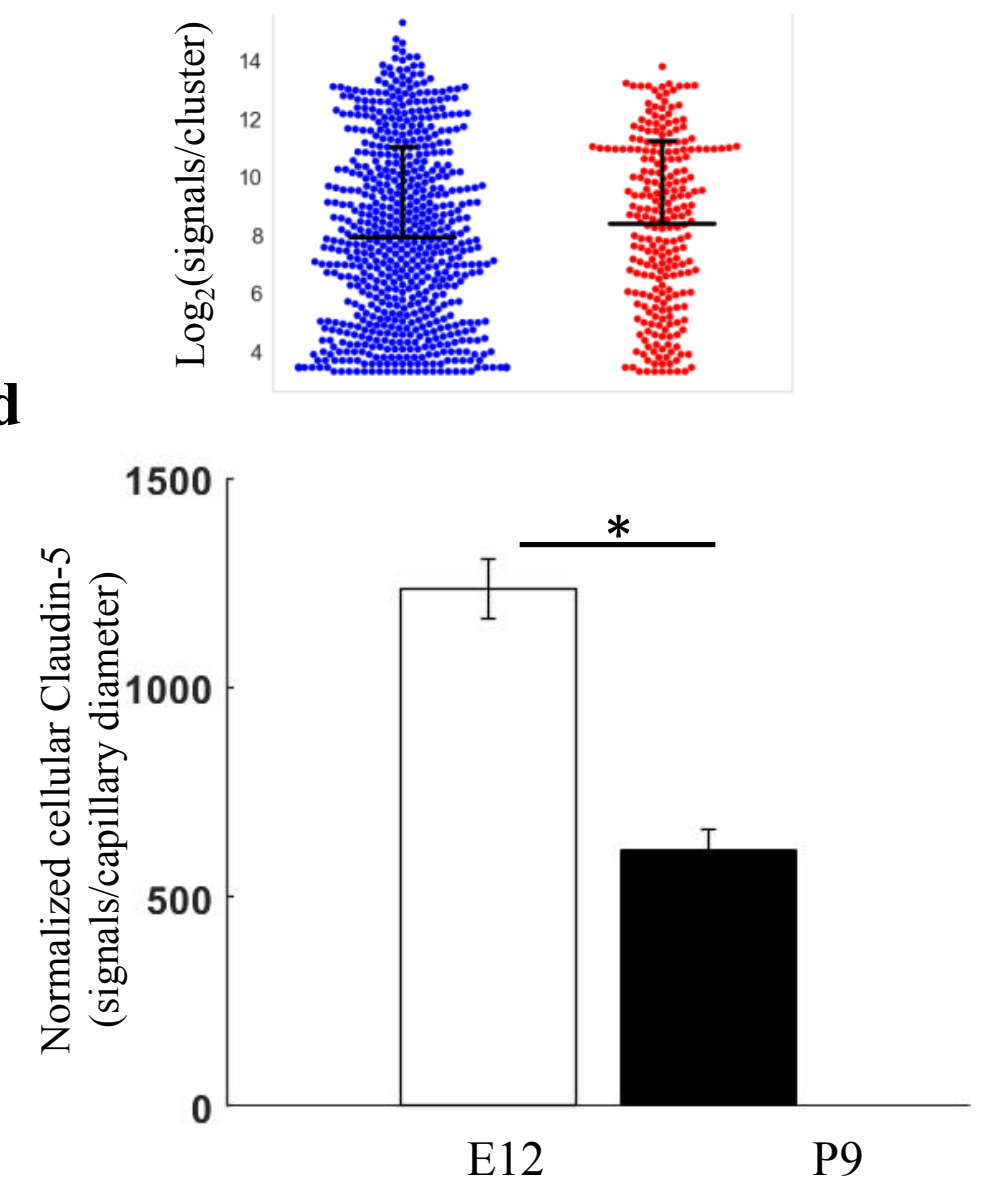


\section{Fig. 5: Total cellular claudin-5 abundance does not correlate with BBB restrictive}

254 properties. Quantifications of claudin-5 levels and clustering properties along developmental

255 BBB maturation a, dSTORM (right) compared to epi-fluorescent images (left) of claudin-5

256 immunostaining in E12 and P9 cortical capillary cross-sections. Two distinct claudin-5

257 organizations could be observed; discrete clusters (arrowheads) and a more defused claudin-5

258 appearance composed of many small clusters with relatively small gaps between them,

259 evident only in E12 capillaries (arrow, see Extended Data Fig. S1a for further analysis). Scale

260 bars, $1 \mu \mathrm{m}$. b, Claudin-5 clustering-properties analysis showed that there were about 2.6 times

261 more discrete clusters per capillary at E12 than at P9 (a set of 20 capillaries of each age).

262 Distribution of claudin-5 clusters area in E12 capillaries was skewed towards smaller clusters

263 and the average cluster area was slightly smaller in E12 with no dramatic difference between

264 the distributions (average of $0.309 \mu \mathrm{m}^{2}$ (E12) vs. $0.3413 \mu \mathrm{m}^{2}$ (P9). There was no dramatic

265 difference in the distribution of signals per cluster or signal densities between the two groups

266 (see Fig. S1b). $n=3$ pups/embryos, 20 capillaries, 657 clusters (E12) and 246 clusters (P9).

267 Data are mean \pm s.e.m. c, Quantifications of total cellular claudin-5 per capillary cross section

268 shows a shift towards lower claudin-5 levels and smaller capillary diameter in P9 than in E12

269 vasculature. Capillary diameter was significantly smaller $(5.9 \pm 0.39 \mu \mathrm{m}(\mathrm{P} 9), 11.1 \pm 0.47 \mu \mathrm{m}$

270 (E12)), total number of claudin-5 signals per capillary was significantly lower $(3,590 \pm 372$

271 (P9), 14,341 $\pm 1,257$ (E12)). d, Normalizing the total number of signals per capillary to its

272 diameter shows the average claudin-5 cellular abundance is significantly lower at P9. $n=25$

273 capillaries (E12) and 27 capillaries (P9) of 3 embryos/pups. Data are mean \pm s.e.m. $* \mathrm{P}<0.05$

274 (Two tailed Mann-Whitney U-test). 
We then expanded the structural and organizational properties examination to include additional TJ proteins in cortical capillaries. Imaging ZO1 and occludin showed that like

277 claudin-5, ZO1 had clustered organization (Fig. 6a left), whereas occludin was much less

278 organized in discrete clusters and had more dispersed organization patterns (Fig. 6a right).

279 Based on published biochemical studies, ZO1 is known to physically interact with the C-

280 terminals of both claudin-5 and occludin, which aligns with our imaging data demonstrating 281 signals of all three in close proximity (Fig. 6, S3).

While each capillary cross-section presented multiple claudin-5 clusters, we assumed that not all claudin-5 proteins are localized to TJs. Indeed we could detect claudin-5 clusters in

284 close proximity with a lysosomal marker (LAMP1, Fig. S4a), suggesting lysosome 285 localization. In addition, we could detect claudin-5 clusters in close proximity with an ER marker (BiP, Fig. S4b), suggesting ER localization. Therefore we focused on structures where claudin-5 and ZO1 clusters were coupled (Fig. 6b arrows), reasoning that these might better reflect actual TJs. We analyzed the density of claudin-5 signals in clusters that were coupled with ZO1 clusters and compared it to the density of claudin-5 signals in independent clusters 290 (Fig. 6b, arrowhead) of both E12 and P9 (Fig. 6b,c). The average density of P9 claudin-5 291 clusters that were coupled with ZO1 clusters was about five-fold higher $(\mathrm{P}<0.0215)$ than in 292 independent claudin-5 clusters. The average density of E12 claudin-5 clusters was similar 293 regardless of proximity to ZO1 clusters, and was low compared to P9 independent claudin-5 294 clusters. We concluded that claudin-5 clusters in TJs (based on pairing with ZO1) have higher 295 claudin-5 density in late developmental stages, a structural feature that correlates with BBB maturation. 
Figure 6

a
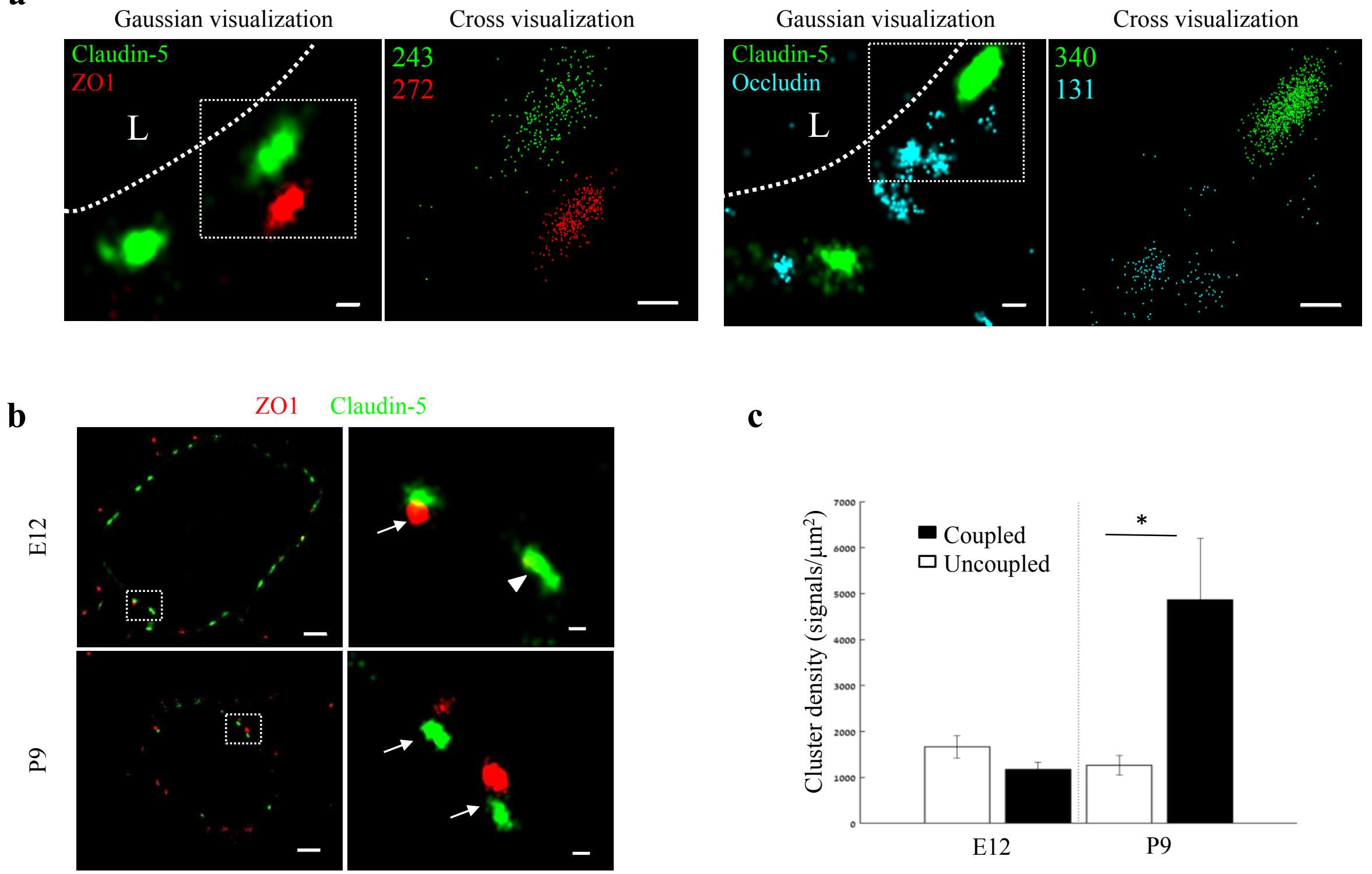

c

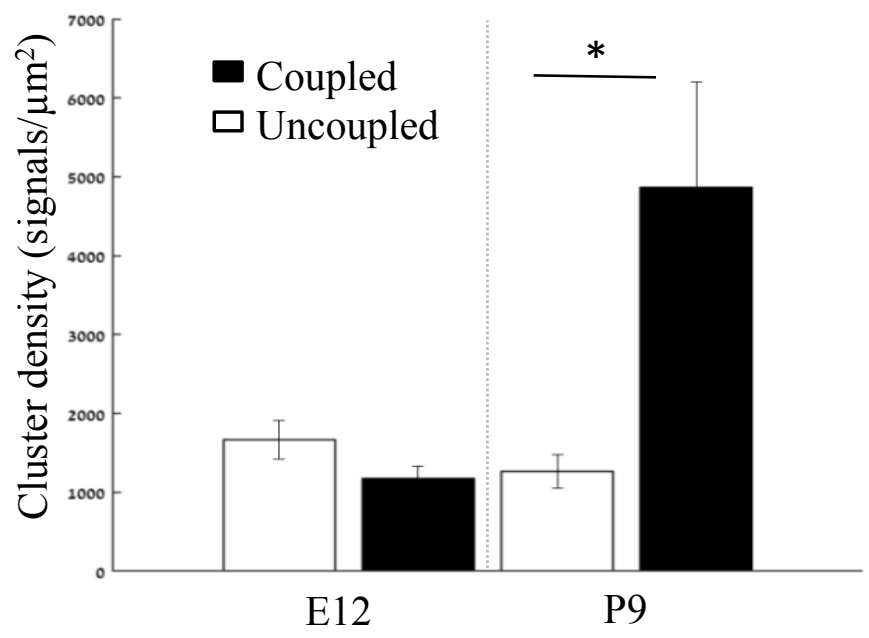


297 Fig. 6: Molecular organization of mouse cortical BBB TJs. Nano-scale molecular organization of TJ proteins in cortical capillaries of postnatal wild-type mice (P9). a, Claudin-

2995 and ZO1 display clustered organization (left) whereas occludin was much less organized in 300 discrete clusters and had more dispersed organization patterns (right). 2D-STORM imaging

301 data demonstrates that signals of all three TJ proteins are in close proximity ('Gaussian

302 visualization' in which signal intensity correlates with localization precision). An inset with 303 magnifications of each cluster (right images) demonstrates the very high molecular density of

304 TJ proteins ('Cross visualization' shows all resolved signals where each single-molecule

305 signal displays as a cross). Scale bars, $100 \mathrm{~nm}$. Representative signal numbers are shown.

$306 n=40$ capillaries of 4 wild-type pups. L - capillary lumen. b, dSTORM imaging (Gaussian

307 visualization) of claudin-5 (green) and ZO1 (red) immunostaining of E12 and P9 cortical 308 capillary cross-sections. Note that some claudin-5 clusters are coupled with ZO1 clusters

309 (high magnification insets, arrows) while some are independent claudin-5 clusters

310 (arrowhead). Scale bars, $1 \mu \mathrm{m}$ and $100 \mathrm{~nm}$ in insets. c, Average density of P9 claudin-5

311 clusters that were coupled with ZO1 clusters was $\sim 5$-fold higher than independent claudin-5

312 clusters. The average density of E12 claudin-5 clusters was similar regardless of proximity to 313 ZO1 clusters, and was low compared to P9 independent claudin-5 clusters. $n=40$ clusters from 31411 capillaries and 43 clusters from 11 capillaries (of 3 embryos/pups, P9 and E12

315 respectively). Data are mean \pm s.e.m. $* \mathrm{P}<0.05$ (Two tailed Mann-Whitney U-test). 
In order to gain insights on mechanisms underlying Nano-scale molecular architecture of

318 BBB TJs, we imaged claudin-5 null and wild-type littermates cortical capillaries with 319 dSTORM. This approach was intended to enable testing whether claudin-5 being a very 320 abundant transmembrane TJ component might be an organizer of other TJ components. We 321 initially confirmed the specificity of claudin-5 antibodies, with no detectable staining in null 322 tissue (confocal microscopy of E16 cortical null and wild-type littermates' tissues, Fig. 7a). 323 Specificity was also confirmed with dSTORM imaging (Fig. 7b). Finally, we found that 324 absence of claudin-5 did not altered, ZO1 clustering organization nor it had any effect on 325 occludin dispersed organization patterns (Fig 7c). In contrary to the previous conclusions of 326 unaltered molecular composition of claudin-5 null TJs (Nitta et al., 2003), quantification of 327 dSTORM imaging revealed that the total cellular occludin levels (normalized to capillary 328 circumference) were higher in claudin-5 null capillaries by $\sim 1.29$ fold compared to occludin 329 levels in wild-type capillaries $(\mathrm{P}<0.0039$, Fig. $7 \mathrm{~d})$. Overall cellular ZO1 expression levels 330 were also higher in claudin-5 null capillaries (not statistically significant). These new findings 331 of molecular alterations in TJ protein levels in the claudin-5 null BBB demonstrate the high 332 sensitivity provided by single molecule super resolution imaging, with these molecular 333 changes most probably obscured when tested by other approaches. We concluded that nano334 scale organization of both ZO1 and occludin are independent of claudin-5 expression (at least 335 in the embryonic setting). We also believe that these findings warrant a new evaluation of 336 claudin-5 function at BBB junctions. 
Figure 7

a

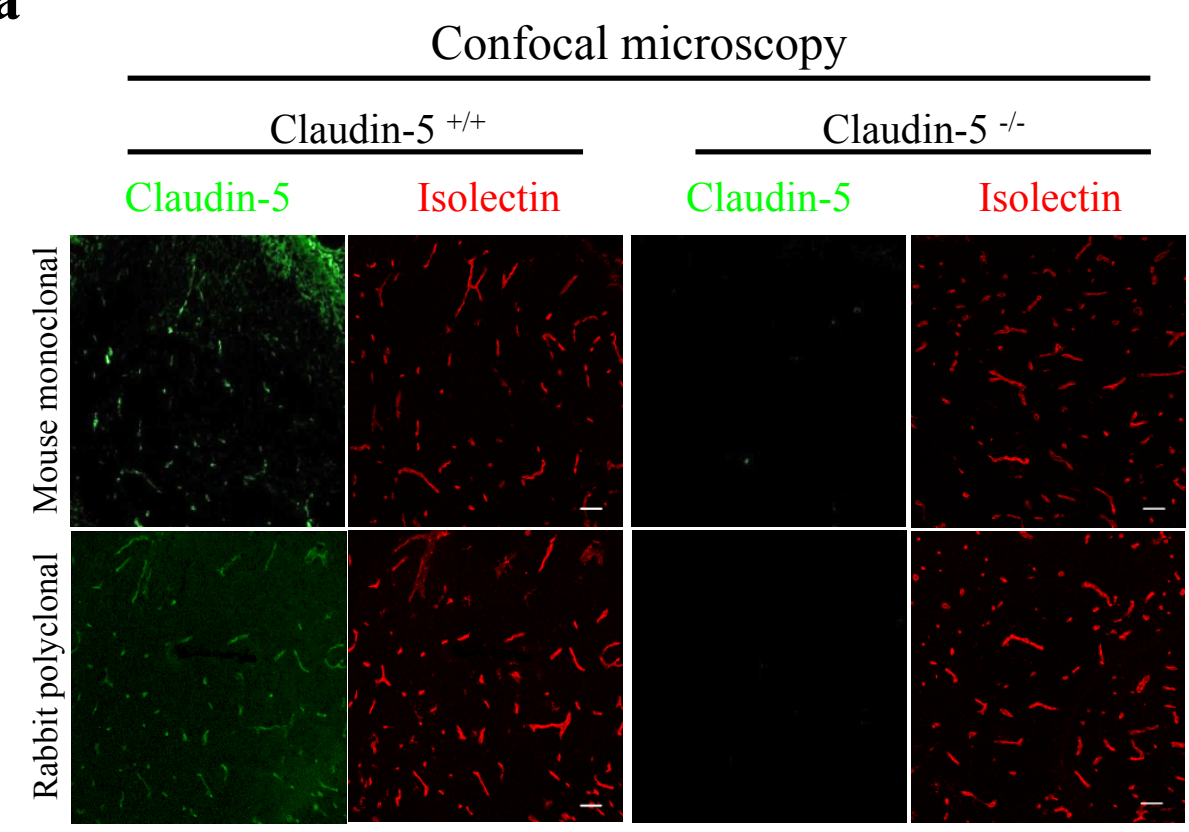

c

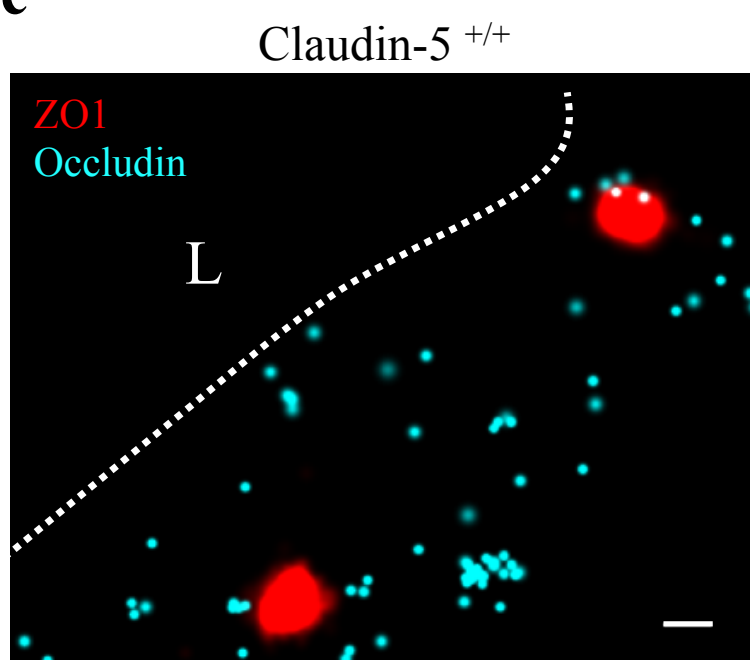

b

STORM microscopy
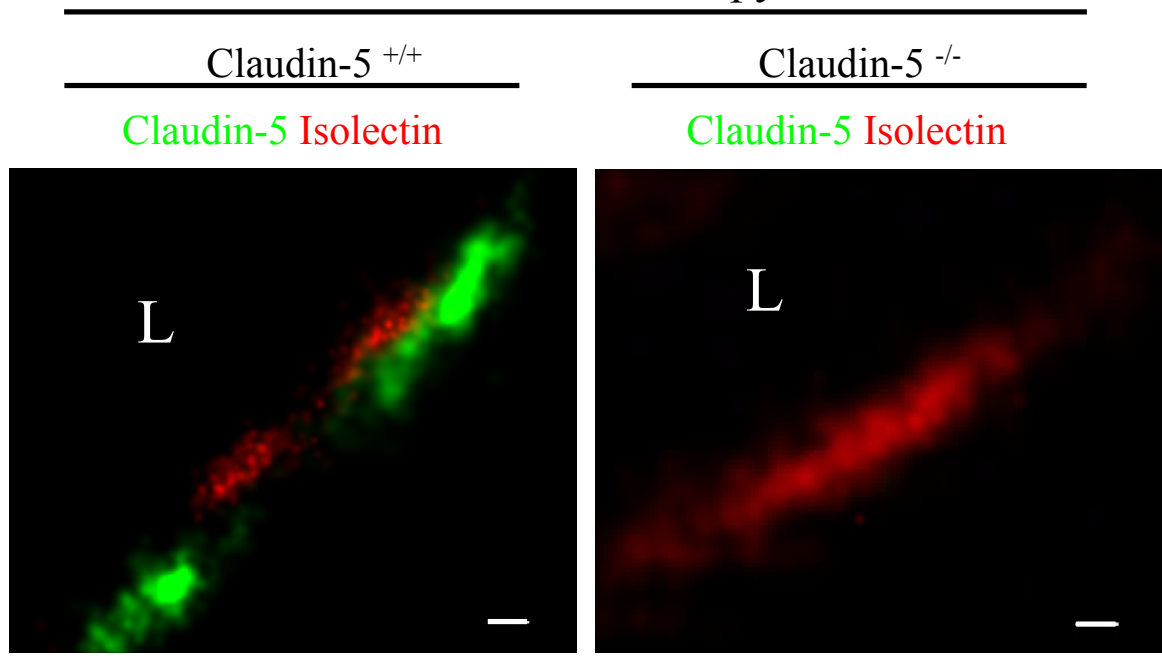

d

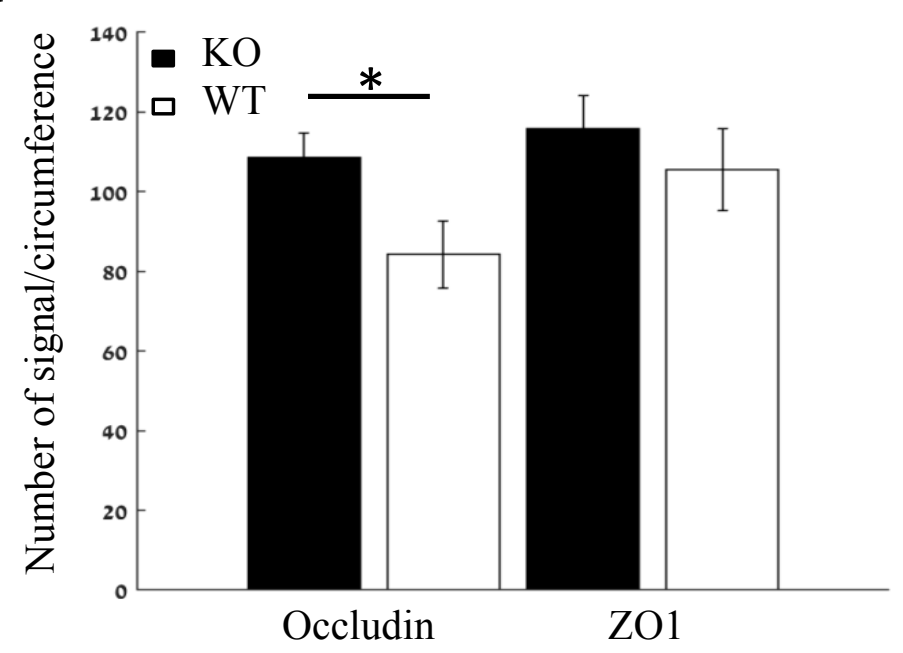



staining in E16 cortical null tissues (Isolectin staining used to localize vasculature, $n=4$ wildtype, 4 claudin-5 null embryos). c, E16 claudin-5 null and wild-type littermates cortical

342 capillaries imaged with dSTORM display unaltered ZO1 clustering organization and occludin 343 dispersed organization patterns. Scale bars, $100 \mathrm{~nm}$. d, Total cellular signal quantifications

344 revealed that occludin levels were $\sim 1.29$-fold higher in claudin-5 null capillaries compared to 345 wild-type. Total cellular ZO1 signal levels were also higher in claudin-5 null capillaries (not 346 statistically significant). Data are mean \pm s.e.m. $* \mathrm{P}<0.05$ (Two tailed Mann-Whitney U-test).

347 L - capillary lumen. $n=109$ capillaries of 4 wild-type embryos and 86 capillaries of 4 claudin3485 null embryos.

\section{Investigating BBB TJ function using super-resolution microscopy}

In light of the identified maturation changes in claudin-5 clustering properties, we sought

to directly test $\mathrm{TJ}$ function in vivo. To this end we employed tracer challenges and compared

353 TJs permeability at three developmental time points (E12, E16 and P9, Fig. 8). We performed dSTORM imaging of cortical capillaries following injection of fluorescent tracers to the blood stream and used claudin-5 immunofluorescence to demarcate capillary boundaries and localize TJs in cortical tissues. Similar to the traditional HRP/EM approach (Reese \&

357 Karnovsky, 1967), dSTORM imaging enables detection of functional BBB TJs with the 358 added value of TJ protein visualization and localization relative to tracer molecules. The $359 \mathrm{HRP} / \mathrm{EM}$ approach is not compatible in young embryos and therefore until now, dysfunction 360 of immature TJs was only speculated to underlie capillary hyper-permeability at early stages 361 of BBB development. Thus we tested E12 TJ function with an in utero embryonic liver tracer 
362 injection method that we previously developed to assess BBB permeability during early 363 mouse developmental stages (Ben-Zvi et al., 2014). As expected from previous experiments 364 with conventional microscopy (Ben-Zvi et al., 2014), with dSTORM imaging we could confirm that E12 capillaries did not restrict movement of tracer molecules across the BBB

366 (Fig. 8a; 10-kDa dextran, upper left. 443 Dalton sulfo-NHS-biotin, lower left). Tracer 367 signals could be detected in the basal side or further away, presumably in brain tissue (Fig. 8, 368 arrowheads). Moreover, we could directly image TJs contribution to this leakage; tracer 369 signals were found intermingled with claudin-5 clusters (Fig. 8, arrows) and in many cases we 370 could detect tracer signals in three locations relative to claudin-5 clusters: the luminal side, 371 the cluster area itself and the abluminal side. We interpreted these as direct evidence of tracer 372 leakage across immature TJs. As expected, mature P9 capillaries restrict movement of the 373 vast majority of tracer molecules (Fig. 8; 10-kDa dextran, upper right. 443 Dalton sulfo374 NHS-biotin, lower right) from the lumen to the basal side (similar results were obtained using 375 Biocytin, data not shown).

In order to examine TJ function along the developmental axis, we also imaged E16 TJs.

377 First, we validated that at E16 cortical TJs were indeed restrictive to the $10-\mathrm{kDa}$ tracers 378 (aligned with general permeability that we previously demonstrated (Ben-Zvi et al., 2014)). 379 TJs at this stage were functional and prevented tracer leakage across claudin-5 clusters (Fig. $3808 \mathrm{a}$, middle-upper). Surprisingly, the smaller tracer, sulfo-NHS-biotin, was not restricted to the 381 vessels' lumen, and was evident also on the brain side (Fig. 8a middle-lower, arrowheads). 382 Therefore at this stage TJs were not as mature as P9 TJs and did not prevent smaller tracer 383 leakage across claudin-5 clusters. These developmental changes in permeability are reflected 384 in quantification of tracer signal density at the abluminal side of the junctions (Fig. 8b,c). We 
bioRxiv preprint doi: https://doi.org/10.1101/2021.08.12.456150; this version posted August 13, 2021. The copyright holder for this preprint (which was not certified by peer review) is the author/funder. All rights reserved. No reuse allowed without permission.

386 selectivity properties and that the dSTORM approach is suitable for in depth investigations of

387 this phenomena in future studies. 
Figure 8

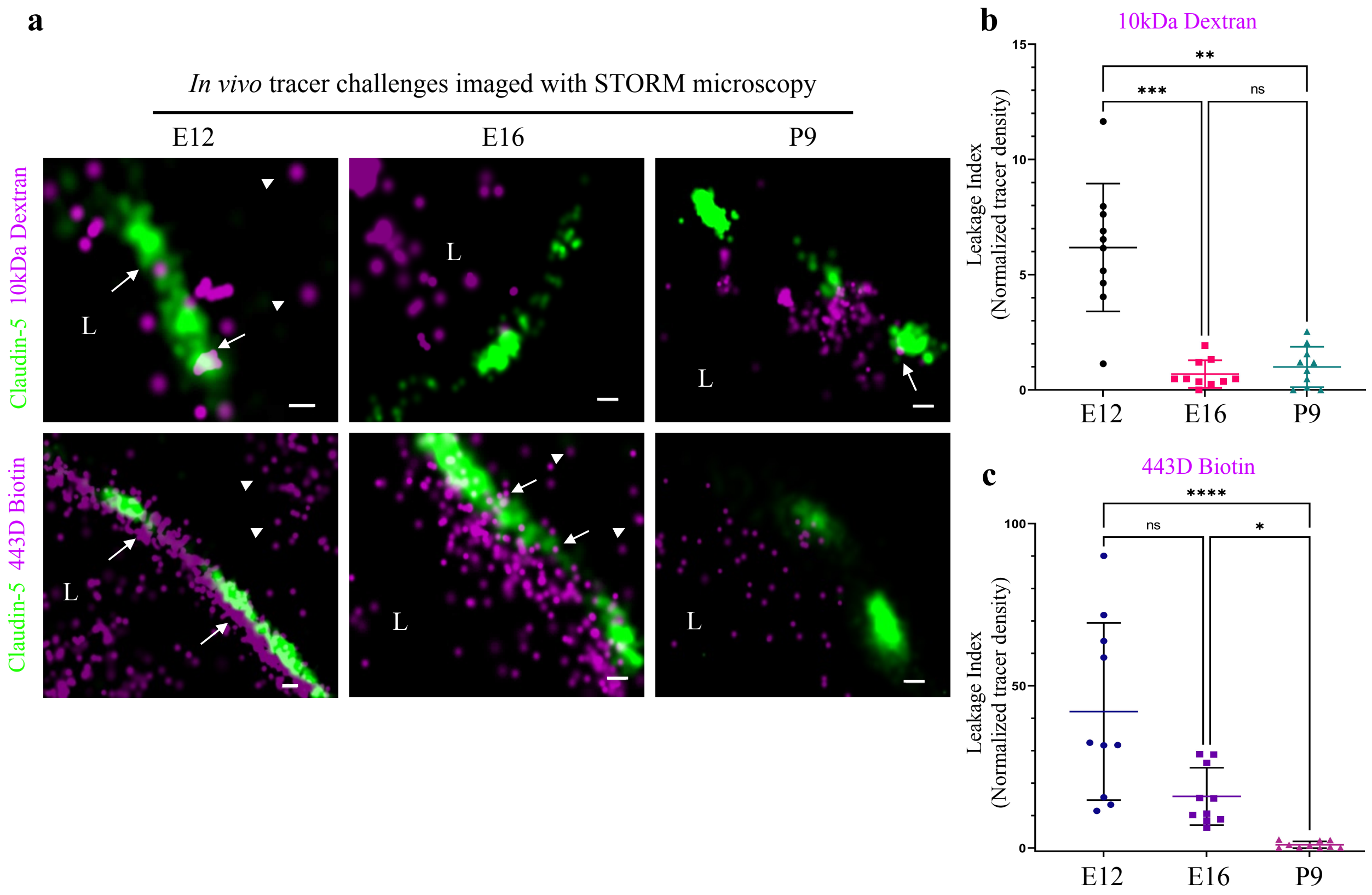


Fig. 8: Investigating BBB TJ function using super-resolution microscopy. Tracer

challenges testing cortical capillaries permeability with dSTORM imaging, provides evidence of leakage across immature TJs. a, E12 TJ function tested with an in utero embryonic liver

391 tracer injection method (Ben-Zvi et al., 2014). $10 \mathrm{kDa}$ dextran signals (upper-left) and $\sim 43$

392 Dalton sulfo-NHS-biotin signals (lower-left) were found in the luminal side, intermingling

393 with claudin-5 clusters (arrows) and in the abluminal side and further away (presumably brain

394 tissue, arrowheads). These were interpreted as evidence of tracer leakage across immature

395 TJs. Following trans-cardiac tracer challenges, dSTORM imaging shows P9 capillaries can

396 restrict movement of tracer molecules from the lumen to the brain side (10 kDa dextran

397 upper-right, and $\sim 43$ Dalton sulfo-NHS-biotin lower-right). E16 cortical capillaries were

398 previously found to prevent leakage of 10-kDa tracers (Ben-Zvi et al., 2014; Licht, Dor-

399 Wollman, Ben-Zvi, Rothe, \& Keshet, 2015), also validated here with dSTORM imaging

400 (middle-upper). Surprisingly, at this stage sulfo-NHS-biotin was not restricted to the vessels'

401 lumen, evident also on the brain side (middle-lower arrowheads) and intermingling with

402 claudin-5 clusters (arrows). Scale bars, $100 \mathrm{~nm}$. L - capillary lumen. $n=40$ capillaries for each

403 tracer of 3 pups/embryos. b-c, Developmental changes in permeability are reflected in

404 quantification of tracer signal density at the abluminal side of the junctions $(10 \mathrm{kDa}$ dextran at

405 b, and 443 Dalton sulfo-NHS-biotin at c). Relative leakage index was calculated as tracer

406 signal density (signals/area) in a fixed area and distance from the abluminal side of the

407 claudin-5 signal, and was normalized to the average signal density at P9 (set as leakage

408 index =1). $n=10$ capillaries for each tracer of 3 pups/embryos, ${ }^{*} \mathrm{P}<0.0123,{ }^{*} * \mathrm{P}<0.0031$

$409 \quad,{ }^{* * *} \mathrm{P}<0.0006,{ }^{*} * * * \mathrm{P}<0.0001$, Kruskal-Wallis test and Dunn's test for multiple comparisons. 


\section{Discussion}

411 We present here a new super resolution imaging approach for BBB research. Using this 412 approach we revealed novel structural and functional features of BBB TJs through 413 examination of TJ maturation of EC cultures, normal cortical mouse BBB development and

414 claudin-5 null embryos. With super-resolution imaging we could overcome the limitations of 415 conventional microscopy, which is essential for BBB TJ investigations since it provides 416 proper spatial resolution and enables sensitive quantifications of $\mathrm{TJ}$ proteins and tracer 417 molecules.

We provide direct evidence of non-functional TJs in the early embryonic BBB. HRP-EM approaches were not useful for studying early embryonic stages; consequently direct evidence 420 for the cellular pathway mediating leakage in early embryogenesis was missing. A recent 421 mouse retina-vasculature study showed that developmentally, the TJ pathway is already restrictive before the vesicular pathway is blocked (Chow \& Gu, 2017) and studies in sheep

423 demonstrated similar trends in the brain (Dziegielewska et al., 1979). We suggest that in 424 mouse cortical vasculature, the course of barrier-genesis might be different. We found that 425 there are different $\mathrm{TJ}$ maturation developmental time-courses for different size selectivities. 426 Thus, even in later stages (E16), when vesicular activity is clearly diminished at the BBB 427 (based on ultra-structural EM studies (Bauer et al., 1993)), we found that TJs were not fully 428 mature and mediate leakage of a low molecular-weight tracer.

429 We focused on three major TJs components and found sparse occluding vs. clustered 430 ZO1/claudin-5 molecular architecture. Since our current study did not include additional TJ 431 components, we could not differentiate in our analyses between subtypes of TJs and therefore 432 these might represent claudin-5 presumably localized to either bicellular or tricellular 433 junctions. 
TJ claudin-5 shifted into denser cluster organization along with in vivo BBB maturation,

435 a finding which is in line with freeze-fracture EM data that suggested cluster organization of

436 EC TJs (Haseloff et al., 2015; Morita et al., 1999). Therefore, we suggest that confocal

437 imaging description of claudin-5 strands might be a misrepresentation of actual claudin-5

438 organization. Notably, dSTORM might give rise to some artificial clustering due to antibody

439 staining and fluorophore overcounting (Burgert, Letschert, Doose, \& Sauer, 2015). Hence,

440 our clustering results of claudin-5 and $\mathrm{ZO} 1$ should be taken as relative changes in protein

441 organization under the different tested conditions. In addition, our imaging included multiple

442 stains (two different antibodies used for claudin-5 and testing several different fluorophores

443 conjugated to secondary antibody combinations, all resulted in similar clustering data).

444 Moreover, occludin did not show similar clustering properties and finally, we used algorithms

445 to minimize fluorophore overcounting (see Methods (Ovesny, Krizek, Borkovec, Svindrych,

446 \& Hagen, 2014)).

447 Previous TJ studies primarily investigated epithelial cells, which have considerably larger

448 volumes than ECs. These studies emphasized a contentious organization of transmembrane

449 proteins along the apical circumference of the cells, providing 'sealing belts'. Our finding of

450 disrupted lines with discrete clusters, forming bead-like structures does not support the

451 concept that claudin-5 fully construct these 'sealing belts'. We can think of three possible

452 explanations for this discrepancy; first, we cannot exclude that our staining approach

453 underrepresents the entire claudin-5 localizations (highly compacted foci might reduce

454 antibodies accessibility). Second, TJ cleft directionality is variable especially in vivo and the

$4553 \mathrm{D}$ organization of the 'sealing belt' might be missed once imaged in $2 \mathrm{D}$. This explanation is

456 incomplete especially once examining the in vitro data where very flat coordinated orientation

457 monolayers are imaged at the cell-glass contacts. Third and most exciting possibility is that

458 other TJ proteins (yet to be discovered) occupy the gaps between claudin-5 clusters; these 
459

460

461

462

463

464

465

466

467

468

469

470

471

472

473

474

475

476

might be coordinately localized to complete the 'sealing belts'. Such theory aligns with the findings that the claudin-5 null BBB has only partially perturbed sealing properties (Nitta et al., 2003).

Our data supports the huge cellular claudin-5 abundance reported by other approaches (e.g average of $\sim 12,000$ transcripts per capillary endothelial cell (Vanlandewijck et al., 2018), average of $\sim 14,000$ (E12) or $\sim 3500$ (P9) signals per cross section in our data). Total levels of junctional proteins/transcripts are presented in many studies (measured by western blot, conventional imaging, qPCR/RNAseq etc. (Armulik et al., 2010; Daneman et al., 2010; Nitta et al., 2003; Vanlandewijck et al., 2018; Y. Zhang et al., 2014)), and down regulation of these components are often used as an indicator for TJ dysfunction (Alvarez et al., 2011; Bell et al., 2010; Bell et al., 2012; Zhong et al., 2008). Our findings that claudin-5 expression inversely correlates with $\mathrm{BBB}$ tightness in the developmental setting might be distinct from the situation in the adult BBB. Similar to our findings, dSTORM imaging of cultured alveolar epithelial cell TJs, shows increase claudin-5 in response to alcohol exposure together with an increase in paracellular leak (Schlingmann et al., 2016). In pathological settings a minimal threshold of claudin-5 expression might result in TJ leakage. Thus, it will be interesting to test if such a threshold is breached in disease (as suggested for the 22q11 syndrome (Greene et al., 2018)).

The differential size selectivity we found in E16 was reminiscent of the seminal study by Nitta et al. (Nitta et al., 2003) demonstrating that the BBB of E18.5 claudin-5 null mouse embryos was hyper-permeable to Hoechst (562 Dalton) and to gadolinium ( 742 D) but not to $10 \mathrm{KDa}$ dextran or to endogenous albumin $(\sim 66 \mathrm{KDa})$. Following this study, claudin-5 was considered the molecular component of $\mathrm{BBB}$ TJs responsible for restricting passage of low molecular weight substances $(<800 \mathrm{D})$. Our data indicates that despite the presence of claudin-5, TJs of E16 wild-type mice display similar differential size selectivity as E18.5 
484 claudin-5 null TJs. Accordingly it seems that claudin-5 is not the sole component responsible

485 for restricting passage of low molecular weight substances.

Further investigations are needed in order to evaluate the possible redundancy between different BBB TJ proteins. The increase in occludin expression we describe for claudin-5 null TJ could reflect a developmental compensatory mechanism that might obscure the full contribution of claudin-5 to TJ function. Nitta et al. also suggested that in the absence of claudin-5, claudin-12-based TJs in brain vessels would function as a molecular sieve restricting high molecular weight substances but allowing low molecular weight substances $(<800 \mathrm{kDa})$ to leak into the brain. Recent RNAseq data indicated low expression of claudin12 in BBB endothelium (approximately 80 fold lower then claudin-5 (Vanlandewijck et al., 2018; Y. Zhang et al., 2014)), reports farther corroborated by a claudin-12 reporter mouse study indicating expression in many other CNS cell types (Castro Dias et al., 2019). compartments via phase separation that can drive TJ formation in epithelia cells (Beutel,

500 Maraspini, Pombo-Garcia, Martin-Lemaitre, \& Honigmann, 2019). It also aligns with 501 claudin-5 null embryos having no overt morphological abnormalities (Nitta et al., 2003). The 502 other two $\mathrm{ZO}$ proteins might also participate in formatting $\mathrm{TJ}$ architecture. Based on 503 scRNAseq data, ZO3 is not expressed at the BBB but ZO2 has lower but significant mRNA 504 levels (Vanlandewijck et al., 2018). It would be interesting to explore ZO2 nano-scale 505 organization as it shares the same self-organization capacity as ZO1 and therefore might display similar clustering properties (Beutel et al., 2019).

507 Further development of the BBB dSTORM approach will provide additional insights: the 508 ability to image proteins together with membrane lipids and 3D reconstruction of super- 
509 resolution imaging are both important focus points for future studies. Deciphering BBB TJ

510 biology with this new approach will aid in evaluating the potential of TJ manipulation for

511 drug delivery and in identifying TJ abnormalities in disease.

513 Methods

514 Animals. ICR (CD-1®, Envigo, Rehovot, Israel) mice were used for embryonic and post515 natal BBB functionality assays and dSTORM imaging. Pregnant mice were obtained 516 following overnight mating (day of vaginal plug is defined as embryonic day 0.5). All 517 animals were housed in SPF conditions and treated according to institutional guidelines 518 approved by the Institutional Animal Care and Use Committee (IACUC) at Hebrew 519 University.

520 The claudin-5 mutant mice (Nitta et al., 2003) were kindly provided by Dr. Mikio Furuse 521 (National Institute for Physiological Sciences, Japan). Mice were housed in individually 522 ventilated cages under specific pathogen-free conditions at $22{ }^{\circ} \mathrm{C}$ with free access to chow and 523 water. E16 claudin-5 wild-type and null embryos were obtained according to procedures 524 approved by the Veterinary Office of the Canton Bern, Switzerland. Claudin-5 null mutant 525 and wild-type embryos were genotyped using lysates prepared from tips of tails using the 526 following 3 PCR primers: Cldn5_UPS: GCCCCTACTAGGACAGAAACTGGTAG; 527 Cldn5_REV1: CAGACCCAGAATTTCCAACGCTGC and PGK-pA-FW1: 528 GCCTGCTCTTTACTGAAGGCTCTT, which provide a 422 bp product for the claudin-5 529 wild-type allele and a $630 \mathrm{bp}$ product for the claudin-5-knockout allele. PCR cycling 530 conditions were: 4 min. $94^{\circ} \mathrm{C} ; 1 \mathrm{~min}$. each at $94^{\circ} \mathrm{C}, 64^{\circ} \mathrm{C}$ and $72^{\circ} \mathrm{C}$; repeated 35 times and a 531 final 5 min elongation step at $72^{\circ} \mathrm{C}$.

532 Tissue preparation. After dissection, brains were placed in 4\% paraformaldehyde (PFA, 533 Sigma Aldrich) at $4{ }^{\circ} \mathrm{C}$ overnight, cryopreserved in $30 \%$ sucrose and frozen in TissueTek 
534 OCT (Sakura). Frozen brains were cut to 5-8 $\mu \mathrm{m}$ slices for immunofluorescent staining

535 (CM1950, Leica) to produce coronal brain sections.

536 Immunohistochemistry. Tissue sections or cell cultures were blocked with $20 \%$ goat serum 537 and $20 \%$ horse serum, permeabilized with $0.5 \%$ Triton X-100, and stained with primary and 538 secondary antibodies (see antibodies table for details). Sample were mounted with freshly 539 made imaging buffer for ASTORM (describe in the dSTORM imaging section) and visualized

540 by dSTORM and epifluorescence, or mounted in Fluoromount G (EMS) and visualized by 541 confocal microscopy. Both a polyclonal and a monoclonal anti-claudin-5 antibody were found 542 to be highly specific in dSTORM, validated with claudin-5 null mice staining, as in confocal 543 imaging (Fig. 7a,b).

\begin{tabular}{|c|c|c|c|c|c|}
\hline Epitope & Class & Host & $\begin{array}{c}\text { Catalogue } \\
\text { number }\end{array}$ & Company & Dilution \\
\hline * Claudin 5 & Monoclonal & Mouse & $35-2500$ & Life Technologies & $1: 100$ \\
\hline $\begin{array}{c}* * \text { Claudin } \\
5\end{array}$ & Polyclonal & Rabbit & 160034 & Zymed & $1: 50$ \\
\hline ZO1/TJP1 & Polyclonal & Rabbit & $61-7300$ & $\begin{array}{c}\text { Thermo Fisher } \\
\text { Scientific }\end{array}$ & $1: 200$ \\
\hline Occludin & Monoclonal & Mouse & $33-1500$ & $\begin{array}{c}\text { Thermo Fisher } \\
\text { Scientific }\end{array}$ & $1: 50$ \\
\hline ERG & Monoclonal & $\begin{array}{l}\text { Rabbit } \\
\text { monoclonal }\end{array}$ & $\begin{array}{l}\text { ab92513/ } \\
\text { EPR3864 }\end{array}$ & abcam & $1: 200$ \\
\hline Lamp1 & Monoclonal & Rat & ID4B & $\begin{array}{l}\text { DSHB/ } \\
\text { AB_528127 }\end{array}$ & $1: 200$ \\
\hline $\mathrm{BiP}$ & Monoclonal & Rabbit & $\begin{array}{c}\text { C50B12 } \\
\# 3177\end{array}$ & $\begin{array}{c}\text { Cell Signaling } \\
\text { Technology }\end{array}$ & $1: 100$ \\
\hline GAPDH & Monoclonal & Rabbit & ab181602 & abcam & $1: 400$ \\
\hline
\end{tabular}




\begin{tabular}{|c|c|c|c|c|}
\hline Fluorophore & Isotype & $\begin{array}{c}\text { Catalogue } \\
\text { number }\end{array}$ & Company & Dilution \\
\hline Alexa fluor ${ }^{\circledR} 647$ & Anti-rabbit IgG & $711-605-152$ & Jackson & $1: 1000$ \\
\hline Alexa fluor $® 647$ & Anti-mouse IgG & $711-605-151$ & Jackson & $1: 1000$ \\
\hline Alexa fluor $® 568$ & Anti-rabbit IgG & A11011 & Life Technologies & $1: 1000$ \\
\hline Alexa fluor ${ }^{\circledR} 568$ & Anti-mouse IgG & A1103-1 & Life Technologies & $1: 1000$ \\
\hline Alexa fluor ${ }^{\circledR} 488$ & Anti-mouse IgG & $715-545-151$ & Jackson & $1: 1000$ \\
\hline $\begin{array}{l}\text { Streptavidin Alexa } \\
\text { fluor }{ }^{\circledR} 647\end{array}$ & Biotin & S32357 & Molecular Probes & $1: 800$ \\
\hline Alexa fluor®647 & Anti-rat IgG & $712-605-153$ & Jackson & $1: 1000$ \\
\hline
\end{tabular}

549 Embryonic BBB permeability assay. We used the method we developed and fully described

550 in our previous publication (Ben-Zvi et al., 2014). In brief, dams were deeply anesthetized

551 with ketamine-xylazine i.p. $(8.5 \mathrm{mg} / \mathrm{ml} \mathrm{ketamine,} 1.5 \mathrm{mg} / \mathrm{ml}$ xylazine, in $100 \mu \mathrm{l} \mathrm{saline})$.

552 Embryos were injected with $5 \mu$ l of Dextran, Alexa Fluor®647 anionic fixable (D22914,

553 Molecular Probes, $2 \mathrm{mg} / \mathrm{ml}$ ) or $5 \mu$ l of EZ-Link ${ }^{\mathrm{TM}}$ Sulfo-NHS-Biotin (21217, Thermo Fisher

554 Scientific, $1 \mu \mathrm{g} / 20 \mu \mathrm{l}$ ), while still attached via the umbilical cord to the mother's blood

555 circulation. Taking advantage of the sinusoidal, fenestrated and highly permeable liver

556 vasculature, dye was injected using a Hamilton syringe into the embryonic liver and was

557 taken up into the circulation in a matter of seconds. After 5 min of circulation, embryonic

558 heads were fixed by 4 hours immersion in $4 \%$ PFA at $4{ }^{\circ} \mathrm{C}$, cryopreserved in $30 \%$ sucrose and

559 frozen in TissueTek OCT (Sakura).

560 Postnatal BBB permeability assay. P9 pups were deeply anaesthetized and $10 \mu 1$ of Dextran,

561 Alexa Fluor ${ }^{\circledR} 647$ anionic fixable (D22914, Molecular Probes, 2 mg/ml) or EZ-Link ${ }^{\mathrm{TM}}$ Sulfo-

562 NHS-Biotin (21217, Thermo Fisher Scientific, $1 \mu \mathrm{g} / 20 \mu \mathrm{l}$ ), were injected into the left

563 ventricle with a Hamilton syringe. After $5 \mathrm{~min}$ of circulation, brains were dissected and fixed

564 by immersion in $4 \%$ PFA at $4^{\circ} \mathrm{C}$ overnight, cryopreserved in $30 \%$ sucrose and frozen in 
TissueTek OCT (Sakura).

566 Cell culture. The mouse brain endothelioma cell line (bEnd.3) was purchased from American

567 Type Culture Collection (Manassas, VA, USA). bEnd.3 cells were cultured with Dulbecco's

568 Modified Eagle's medium high glucose (DMEM), supplemented with $10 \%$ fetal bovine serum

569 and 1\% penicillin-streptomycin solution (Biological Industries, Beit HaEmek, Israel). Cells

570 were incubated at $37^{\circ} \mathrm{C}$ in a humid atmosphere in the presence of $5 \% \mathrm{CO} 2$. Cells at passages

571 26-27 were suspended (0.25\% Trypsin EDTA B, Biological Industries) and seeded on $24 \mathrm{~mm}$

572 precision coverslips (no. 1.5H, Marienfeld-superior, Lauda-Königshofen, Germany). Cells

573 were washed with PBS and fixed with 4\% PFA (at indicated time point; up to 7 days or more

574 than 11 days post-confluence).

575 iPSC differentiation to brain microvascular endothelial-like cells (iBMECs). iPSCs from

576 a healthy individual (BGUi012-A) (Falik et al., 2020) were cultured between passages 10-17,

577 seeded on Matrigel (Corning) with daily replacement of NutriStem medium (Biological

578 Industries) as previously described ((Falik et al., 2020), PMID: 32905996). iPSCs were

579 passaged every 6-7 days with Versene (Life Technologies) at a 1:12 ratio. Differentiation into

580 iBMECs was carried out as previously described (Jagadeesan, Workman, Herland, Svendsen,

581 \& Vatine, 2020; Vatine et al., 2017; Vatine et al., 2019); cells were passaged and cultured for

582 2-3 days until reaching a density of $2-3 \times 10^{5}$ cells/well. Next, medium was replaced with 583 unconditioned medium without bFGF [UM/F: $200 \mathrm{~mL}$ of DMEM/F12 (1:1) (Gibco), $50 \mathrm{~mL}$

584 knock-out serum replacement (Gibco), $2.5 \mathrm{~mL}$ non-essential amino acids (Gibco), $1.25 \mathrm{~mL}$ of 585 gluta-max (Gibco), $3.8 \mathrm{uL}$ of $\beta$-mercapto-ethanol (Sigma), and $2.5 \mathrm{~mL}$ PSA (BI)] and changed daily for six days. Medium was then replaced with human endothelial serum-free medium (hESFM, Life Technologies) supplemented with $20 \mathrm{ng} / \mathrm{mL}$ bFGF and $10 \mathrm{mM}$ All588 trans retinoic acid (RA) (Sigma) (Biomedical Technologies, Inc.) for 2 days. Cells were then 589 gently dissociated into single cells with Accutase (StemPro) and plated in hESFM medium at 
590 a density of $1 \times 10^{6}$ cells on transwells $(0.4 \mu \mathrm{m}$ pore size; Corning), coverslips or petri dishes 591 that were pre-coated with a mixture of collagen IV (400 ug/mL; Sigma) and fibronectin (100 $592 \mathrm{ug} / \mathrm{mL}$; Sigma).

593 TEER measurements. Trans-endothelial electrical resistance (TEER) was measured every $59424 \mathrm{~h}$ following iBMEC seeding. Resistance was recorded using an EVOM ohmmeter with 595 STX2 electrodes (World Precision Instruments). TEER values were presented as $\Omega \times c m 2$ 596 following the subtraction of an empty transwell and multiplication by $1.12 \mathrm{~cm}^{2}$ to account for 597 the surface area. TEER measurements were measured three independent times for each 598 sample and at least twice for each experimental condition.

599 Paracellular permeability measurements. Sodium fluorescein $(10 \mathrm{mM})$ was added to the 600 upper chamber of the Transwells. Aliquots $(100 \mu \mathrm{l})$ were collected from the bottom chamber 601 every $15 \mathrm{~min}$ and replaced with fresh medium. Fluorescence (485 nm excitation and $530 \mathrm{~nm}$ 602 emission) was quantified at the end of the experiment with a plate reader. Rate of tracer 603 accumulation was used to calculate Pe values was as previously described (Vatine et al., 604 2017). Monolayer fidelity was confirmed at the beginning and at the end of each experiment 605 by TEER measurements.

606 iBMECs STORM imaging. iBMECs were seeded on $24 \mathrm{~mm}$ precision coverslips (no. 1.5H, 607 Marienfeld-Superior, Lauda-Königshofen, Germany), pre-coated with a mixture of collagen $608 \mathrm{IV}$ (400 ug/mL; Sigma) and fibronectin (100 ug/mL; Sigma). Cultures were fixed in 4\% 609 paraformaldehyde for $20 \mathrm{~min}$ at room temperature (RT), washed three times with PBS and 610 kept in $4^{\circ} \mathrm{C}$ until processing.

611 Western blot analysis. Whole cell extracts were isolated using RIPA buffer (50 mM Tris pH $6127.4,150 \mathrm{mM} \mathrm{NaCl}, 5 \mathrm{mM}$ EDTA $\mathrm{pH}$ 8.0, and 1\% Nonidet-P40) supplemented with protease 613 inhibitors (Roche). The concentration of the isolated proteins was determined using Bradford 
614 reagent (Sigma). 30-50 micrograms of the protein were separated on a $15 \%$ polyacrylamide 615 gel and electrophoretically transferred to PVDF membranes (Millipore). Membranes 616 incubated with the primary antibodies against claudin-5 (1:1000,Zymed 1600-34) or GAPDH 617 (1:400, ab181602, ABCAM)) and the appropriate secondary antibodies.

dSTORM imaging. We used a dSTORM system, which allows imaging at approximately 20 nm resolution by using photo-switchable fluorophores (all dSTORM imaging was done on

620 TIRF mode). $5 \mu \mathrm{m}$ brain slices were mounted on poly-D-lysine coated coverslips (no. 1.5H, 621 Marienfeld-superior, Lauda-Königshofen, Germany). dSTORM imaging was performed in a 622 freshly prepared imaging buffer containing $50 \mathrm{mM}$ Tris $(\mathrm{pH} 8.0), 10 \mathrm{mM} \mathrm{NaCl}$ and $10 \%$ $623(\mathrm{w} / \mathrm{v})$ glucose with an oxygen-scavenging GLOX solution $(0.5 \mathrm{mg} / \mathrm{ml}$ glucose oxidase 624 (Sigma-Aldrich), $40 \mu \mathrm{g} / \mathrm{ml}$ catalase (Sigma-Aldrich), $10 \mathrm{mM}$ cysteamine MEA (Sigma625 Aldrich) and 1\% $\beta$ mercaptoethanol (Barna et al., 2016; Dempsey, Vaughan, Chen, Bates, \& Zhuang, 2011; J. Zhang, Carver, Choveau, \& Shapiro, 2016). A Nikon Ti-E inverted

627 microscope was used. The N-STORM Nikon system was built on TIRF illumination using a 6281.49 NA X100 oil immersion objective and an ANDOR DU-897 camera. 488, 568 and 647 $629 \mathrm{~nm}$ laser lines were used for activation with cycle repeat of $\sim 8000$ cycles for each channel. 630 Nikon NIS Element software was used for acquisition and analysis; analysis was also 631 performed by ThunderSTORM (NIH ImageJ (Ovesny et al., 2014)). Images in 2D were 632 Gaussian fit of each localization; in the N-STORM software.

633 dSTORM quantifications. The dSTORM approach we used is based on labeling the target 634 protein with a primary antibody and then using a secondary antibody conjugated to a 635 fluorophore. Thus resolved signals represent a location that is approximately $40 \mathrm{~nm}$ from the 636 actual epitope (assuming the approximation of the two antibodies' length in a linear 637 conformation). The number of signals represents an amplification of the actual target 638 numbers. Amplification corresponds to the primary antibody in the case of a polyclonal 
639 antibody (assuming binding to several epitopes in the same protein, which could be reduced

640 by the use of monoclonal antibodies). Amplification also corresponds to several secondary

641 antibodies binding to a single primary antibody and to several fluorophores attached to a

642 single secondary antibody. Nevertheless, resolution of approximately $20 \mathrm{~nm}$ allows us to

643 separate signals and to use these as proxies to the abundance of target molecules, which can

644 reliably be used to compare different states.

645 Cellular expression level quantifications: We defined the capillary cross-section as an 646 endothelial unit and quantified claudin-5 signals within capillary cross-sections as proxy to 647 total cellular claudin-5 expression levels.

648 Cluster area, signal numbers and signal densities: Single molecule localization microscopy 649 (SMLM) results in point patterns having specific coordinates of individual detected molecules. These coordinates are typically summarized in a 'molecular list' (provided by 651 ThunderSTORM analysis (NIH ImageJ) (Ovesny et al., 2014)). In order to define molecular 652 clusters, we analyzed the molecular lists through a custom Matlab code (MathWorks) using 653 the Matlab functions "Cluster" and "Linkage", as follows: First, our code calculated distances 654 between each point and all other points in the point pattern of the SMLM image. Then, we set 655 a distance threshold for defining molecules that belong to the same cluster: two points were 656 defined to be clustered if their distance was smaller than the threshold distance (e.g. $70 \mathrm{~nm}$ ). 657 All points that were clustered with a specific point belong to one cluster (as defined by 658 linkage function). Hence, a point could only be within one cluster. The code then defined and 659 saved the properties of each cluster, such as the area of the cluster, the number of points 660 within the cluster, and the number of clusters. Cluster densities were calculated as number of 661 points divided by each cluster area. Finally, the point patterns were visualized, while showing 662 all points that belong to the same cluster with the same identifying color (Fig. 1c, S1). The 70 $663 \mathrm{~nm}$ threshold distance used for quantifications was determined based on the following 
664 parameters: minimal distance could not be below $40 \mathrm{~nm}$ (see above antibody labeling

665 strategy); BBB TJs covering continuous contact points, as we evaluated in published TEM

666 imaging data, range approximately up to $100 \mathrm{~nm}$; simulation of claudin-5 density in clusters,

667 measured in different threshold distances between 50-100 nm did not yield significant

668 differences (Fig. S3).

669 Confocal imaging. Images were captured using Nikon Eclipse Ni confocal microscope, 670 objective X20 with Nikon C2 camera and Nis-Elements software. Images are maximal z671 projection of optical sections taken from a $12 \mu \mathrm{m}$ tissue section imaged with $0.85 \mu \mathrm{m}$ 672 intervals.

673 Epi-Fluorescence microscopy. Images presented in Figures 1a, 4a, were taken using an 674 Olympus BX51, 10X/0.3 and 20X/0.5, with Andor Zyla camera, and Nikon NIS elements 675 software (version D4.5) for both image acquisition and analysis.

676 Statistical analysis. All comparisons were performed by two-tailed Mann-Whitney U-tests, 677 or by two tailed pair $\mathrm{t}$ - test (as indicated in the figure legends), $\mathrm{P}<0.05$ was considered 678 significant (GraphPad Prism 8.0.1(244) for Windows, GraphPad Software, San diego, 679 California, USA). For multiple comparisons of leakage index (Figure 8), the Kruskal-Wallis 680 test and Dunn's tests for multiple comparisons were used. For the comparison between post confluence and super confluence we used cluster densities across experiments, for the comparison between ZO1 paired and unpaired claudin-5 clusters we used cluster densities 683 across experiments, for comparisons related to capillaries diameter and total claudin-5 levels 684 we used capillaries across experiments and for comparisons of total occludin levels in wild685 type and caludin-5 null embryos we used capillaries across experiments (for exact repetitions 686 see figure legends). Sample size for all immunofluorescence experiments was determined 687 empirically using standards generally employed by the field: a minimum of three animals per group in each experiment, a minimum of four tissue sections of each tissue and a minimum of 
10 capillaries per group. In the data set of claudin-5 null and control littermates, the person collecting the data and analyzing was blind to the animal's genotype.

691

692

693

694

695

696

697

698

699

700

701

702

703

704

705

706

707

708

\section{Acknowledgments}

We would like to thank; Mss. Sivan Gelb, Kian Atamny and Victoria Miller of the BenZvi group for scientific and writing inputs, Drs. Avihu Klar and Danny Ben-Zvi for scientific inputs, Dr. Gillian Kay for valuable scientific editing, Dr. Norman Grover for his helpful advice regarding statistical analyses, and Ms. Yaara Arad for help with data presentation. This study was supported by the Leona M. and Harry B. Helmsley Charitable Trust (2015PGISL007); and the Israel Science Foundation (grants 1882/16 and 2402/16) to ABZ, the Swiss National Science Foundation (grant 1890809) to BE and the Israel Science Foundation (grant 1621/18) and the Ministry of Science and Technology, Israel (grant 3-15647) to GDV.

We wish to dedicate this work to the memory of Prof. Morris J. Karnovsky of Harvard Medical School, a scientific giant and pioneer of the BBB research field. We are grateful for his mentorship and scientific support, and especially for encouraging us to pursue our endeavor of super resolution imaging of BBB TJs.

\section{Contribution}

ESa performed the majority of the experiments together with RD, SA, BB, MZ and UD. $\mathrm{ABZ}$ and ESa conceived the project and designed the experimental plan. ESa, SA and ABZ wrote this manuscript, with $\mathrm{BE}, \mathrm{RD}, \mathrm{OY}, \mathrm{ESh}, \mathrm{GDV}$ and $\mathrm{BB}$, reviewing and contributing to writing. OY wrote custom Matlab codes for dSTORM analyses; ESh provided critical scientific guidance for dSTORM imaging and analyses. UD and BE supported claudin-5 null mouse strain experiments. $\mathrm{MZ}$ and $\mathrm{GV}$ preformed $\mathrm{iBMEC}$ experiments. RD supported the development of imaging methods and the establishment of the dSTORM imaging system.

\section{Ethics declarations}

Competing interests 
bioRxiv preprint doi: https://doi.org/10.1101/2021.08.12.456150; this version posted August 13, 2021. The copyright holder for this preprint (which was not certified by peer review) is the author/funder. All rights reserved. No reuse allowed without permission.

714 The authors declare no competing interests.

715 


\section{References}

Alvarez, J. I., Dodelet-Devillers, A., Kebir, H., Ifergan, I., Fabre, P. J., Terouz, S., . . Prat, A. (2011). The Hedgehog pathway promotes blood-brain barrier integrity and CNS immune quiescence. Science, 334(6063), 1727-1731. doi:10.1126/science.1206936

Armulik, A., Genove, G., Mae, M., Nisancioglu, M. H., Wallgard, E., Niaudet, C., . . . Betsholtz, C. (2010). Pericytes regulate the blood-brain barrier. Nature, 468(7323), 557-561. doi:10.1038/nature09522

Barna, L., Dudok, B., Miczan, V., Horvath, A., Laszlo, Z. I., \& Katona, I. (2016). Correlated confocal and super-resolution imaging by VividSTORM. Nat Protoc, 11(1), 163-183. doi:10.1038/nprot.2016.002

Bauer, H. C., Bauer, H., Lametschwandtner, A., Amberger, A., Ruiz, P., \& Steiner, M. (1993). Neovascularization and the appearance of morphological characteristics of the blood-brain barrier in the embryonic mouse central nervous system. Brain Res Dev Brain Res, 75(2), 269-278. doi:10.1016/0165-3806(93)90031-5

Bauer, H. C., Krizbai, I. A., Bauer, H., \& Traweger, A. (2014). "You Shall Not Pass"-tight junctions of the blood brain barrier. Front Neurosci, 8, 392.

Bell, R. D., Winkler, E. A., Sagare, A. P., Singh, I., LaRue, B., Deane, R., \& Zlokovic, B. V. (2010). Pericytes control key neurovascular functions and neuronal phenotype in the adult brain and during brain aging. Neuron, 68(3), 409-427.

Bell, R. D., Winkler, E. A., Singh, I., Sagare, A. P., Deane, R., Wu, Z., . . Zlokovic, B. V. (2012). Apolipoprotein E controls cerebrovascular integrity via cyclophilin A. Nature, 485(7399), 512-516.

Ben-Zvi, A., Lacoste, B., Kur, E., Andreone, B. J., Mayshar, Y., Yan, H., \& Gu, C. (2014). $\mathrm{Mfsd} 2 \mathrm{a}$ is critical for the formation and function of the blood-brain barrier. Nature, 509(7501), 507-511. doi:10.1038/nature13324

Beutel, O., Maraspini, R., Pombo-Garcia, K., Martin-Lemaitre, C., \& Honigmann, A. (2019). Phase Separation of Zonula Occludens Proteins Drives Formation of Tight Junctions. Cell, 179(4), 923-936 e911. doi:10.1016/j.cell.2019.10.011

Brightman, M. W., \& Reese, T. S. (1969). Junctions between intimately apposed cell membranes in the vertebrate brain. $J$ Cell Biol, 40(3), 648-677. doi: $10.1083 /$ jcb.40.3.648

Burgert, A., Letschert, S., Doose, S., \& Sauer, M. (2015). Artifacts in single-molecule localization microscopy. Histochem Cell Biol, 144(2), 123-131. doi:10.1007/s00418$015-1340-4$

Butt, A. M., Jones, H. C., \& Abbott, N. J. (1990). Electrical resistance across the blood-brain barrier in anaesthetized rats: a developmental study. J Physiol, 429, 47-62.

Castro Dias, M., Coisne, C., Baden, P., Enzmann, G., Garrett, L., Becker, L., . . . Engelhardt, B. (2019). Claudin-12 is not required for blood-brain barrier tight junction function. Fluids Barriers CNS, 16(1), 30.

Chow, B. W., \& Gu, C. (2017). Gradual Suppression of Transcytosis Governs Functional Blood-Retinal Barrier Formation. Neuron, 93(6), 1325-1333 e1323.

Daneman, R., Zhou, L., Kebede, A. A., \& Barres, B. A. (2010). Pericytes are required for blood-brain barrier integrity during embryogenesis. Nature, 468(7323), 562-566. doi: $10.1038 /$ nature09513

Dempsey, G. T., Vaughan, J. C., Chen, K. H., Bates, M., \& Zhuang, X. (2011). Evaluation of fluorophores for optimal performance in localization-based super-resolution imaging. Nat Methods, 8(12), 1027-1036. 
Dziegielewska, K. M., Evans, C. A., Malinowska, D. H., Mollgard, K., Reynolds, J. M., Reynolds, M. L., \& Saunders, N. R. (1979). Studies of the development of brain barrier systems to lipid insoluble molecules in fetal sheep. J Physiol, 292, 207-231.

Falik, D., Rabinski, T., Zlotnik, D., Eshel, R., Zorsky, M., Garin-Shkolnik, T., . . Vatine, G. D. (2020). Generation and characterization of iPSC lines (BGUi004-A, BGUi005-A) from two identical twins with polyalanine expansion in the paired-like homeobox $2 \mathrm{~B}$ (PHOX2B) gene. Stem Cell Res, 48, 101955. doi:10.1016/j.scr.2020.101955

Furuse, M., Fujita, K., Hiiragi, T., Fujimoto, K., \& Tsukita, S. (1998). Claudin-1 and -2: novel integral membrane proteins localizing at tight junctions with no sequence similarity to occludin. J Cell Biol, 141(7), 1539-1550.

Furuse, M., Hirase, T., Itoh, M., Nagafuchi, A., Yonemura, S., Tsukita, S., \& Tsukita, S. (1993). Occludin: a novel integral membrane protein localizing at tight junctions. $J$ Cell Biol, 123(6 Pt 2), 1777-1788.

Greene, C., Kealy, J., Humphries, M. M., Gong, Y., Hou, J., Hudson, N., . . Campbell, M. (2018). Dose-dependent expression of claudin-5 is a modifying factor in schizophrenia. Mol Psychiatry, 23(11), 2156-2166.

Hagan, N., \& Ben-Zvi, A. (2015). The molecular, cellular, and morphological components of blood-brain barrier development during embryogenesis. Semin Cell Dev Biol, 38, 715. doi:10.1016/j.semcdb.2014.12.006

Haseloff, R. F., Dithmer, S., Winkler, L., Wolburg, H., \& Blasig, I. E. (2015). Transmembrane proteins of the tight junctions at the blood-brain barrier: structural and functional aspects. Semin Cell Dev Biol, 38, 16-25. doi:10.1016/j.semcdb.2014.11.004

Jagadeesan, S., Workman, M. J., Herland, A., Svendsen, C. N., \& Vatine, G. D. (2020). Generation of a Human iPSC-Based Blood-Brain Barrier Chip. J Vis Exp(157). doi: $10.3791 / 60925$

Kealy, J., Greene, C., \& Campbell, M. (2018). Blood-brain barrier regulation in psychiatric disorders. Neurosci Lett, 133664. doi:10.1016/j.neulet.2018.06.033

Knowland, D., Arac, A., Sekiguchi, K. J., Hsu, M., Lutz, S. E., Perrino, J., . . . Agalliu, D. (2014). Stepwise recruitment of transcellular and paracellular pathways underlies blood-brain barrier breakdown in stroke. Neuron, 82(3), 603-617.

Koto, T., Takubo, K., Ishida, S., Shinoda, H., Inoue, M., Tsubota, K., . . I Ikeda, E. (2007). Hypoxia disrupts the barrier function of neural blood vessels through changes in the expression of claudin-5 in endothelial cells. Am J Pathol, 170(4), 1389-1397.

Langen, U. H., Ayloo, S., \& Gu, C. (2019). Development and Cell Biology of the BloodBrain Barrier. Annu Rev Cell Dev Biol, 35, 591-613. doi:10.1146/annurev-cellbio100617-062608

Licht, T., Dor-Wollman, T., Ben-Zvi, A., Rothe, G., \& Keshet, E. (2015). Vessel maturation schedule determines vulnerability to neuronal injuries of prematurity. $J$ Clin Invest, 125(3), 1319-1328.

Liebner, S., Dijkhuizen, R. M., Reiss, Y., Plate, K. H., Agalliu, D., \& Constantin, G. (2018). Functional morphology of the blood-brain barrier in health and disease. Acta Neuropathol, 135(3), 311-336.

Lippmann, E. S., Al-Ahmad, A., Azarin, S. M., Palecek, S. P., \& Shusta, E. V. (2014). A retinoic acid-enhanced, multicellular human blood-brain barrier model derived from stem cell sources. Sci Rep, 4, 4160. doi:10.1038/srep04160

Lippmann, E. S., Azarin, S. M., Kay, J. E., Nessler, R. A., Wilson, H. K., Al-Ahmad, A., . . . Shusta, E. V. (2012). Derivation of blood-brain barrier endothelial cells from human pluripotent stem cells. Nat Biotechnol, 30(8), 783-791. doi:10.1038/nbt.2247 
Martin-Padura, I., Lostaglio, S., Schneemann, M., Williams, L., Romano, M., Fruscella, P., . . Dejana, E. (1998). Junctional adhesion molecule, a novel member of the immunoglobulin superfamily that distributes at intercellular junctions and modulates monocyte transmigration. J Cell Biol, 142(1), 117-127.

Morita, K., Sasaki, H., Furuse, M., \& Tsukita, S. (1999). Endothelial claudin: claudin5/TMVCF constitutes tight junction strands in endothelial cells. J Cell Biol, 147(1), 185-194.

Nitta, T., Hata, M., Gotoh, S., Seo, Y., Sasaki, H., Hashimoto, N., . . . Tsukita, S. (2003). Size-selective loosening of the blood-brain barrier in claudin-5-deficient mice. $J$ Cell Biol, 161(3), 653-660.

Ovesny, M., Krizek, P., Borkovec, J., Svindrych, Z., \& Hagen, G. M. (2014). ThunderSTORM: a comprehensive ImageJ plug-in for PALM and STORM data analysis and super-resolution imaging. Bioinformatics, 30(16), 2389-2390.

Reese, T. S., \& Karnovsky, M. J. (1967). Fine structural localization of a blood-brain barrier to exogenous peroxidase. J Cell Biol, 34(1), 207-217. doi:10.1083/jcb.34.1.207

Saunders, N. R., Liddelow, S. A., \& Dziegielewska, K. M. (2012). Barrier mechanisms in the developing brain. Front Pharmacol, 3, 46. doi:10.3389/fphar.2012.00046

Schlingmann, B., Overgaard, C. E., Molina, S. A., Lynn, K. S., Mitchell, L. A., Dorsainvil White, S., . . Koval, M. (2016). Regulation of claudin/zonula occludens-1 complexes by hetero-claudin interactions. Nat Commun, 7, 12276.

Sohet, F., Lin, C., Munji, R. N., Lee, S. Y., Ruderisch, N., Soung, A., . . . Daneman, R. (2015). LSR/angulin-1 is a tricellular tight junction protein involved in blood-brain barrier formation. J Cell Biol, 208(6), 703-711.

Sweeney, M. D., Sagare, A. P., \& Zlokovic, B. V. (2018). Blood-brain barrier breakdown in Alzheimer disease and other neurodegenerative disorders. Nat Rev Neurol, 14(3), 133150.

van de Linde, S., Loschberger, A., Klein, T., Heidbreder, M., Wolter, S., Heilemann, M., \& Sauer, M. (2011). Direct stochastic optical reconstruction microscopy with standard fluorescent probes. Nat Protoc, 6(7), 991-1009. doi:10.1038/nprot.2011.336

Vanlandewijck, M., He, L., Mae, M. A., Andrae, J., Ando, K., Del Gaudio, F., . . . Betsholtz, C. (2018). A molecular atlas of cell types and zonation in the brain vasculature. Nature, 554(7693), 475-480. doi:10.1038/nature25739

Vatine, G. D., Al-Ahmad, A., Barriga, B. K., Svendsen, S., Salim, A., Garcia, L., . . . Svendsen, C. N. (2017). Modeling Psychomotor Retardation using iPSCs from MCT8-Deficient Patients Indicates a Prominent Role for the Blood-Brain Barrier. Cell Stem Cell, 20(6), 831-843.e835. doi:10.1016/j.stem.2017.04.002

Vatine, G. D., Barrile, R., Workman, M. J., Sances, S., Barriga, B. K., Rahnama, M., . . . Svendsen, C. N. (2019). Human iPSC-Derived Blood-Brain Barrier Chips Enable Disease Modeling and Personalized Medicine Applications. Cell Stem Cell, 24(6), 995-1005.e1006. doi:10.1016/j.stem.2019.05.011

Zhang, J., Carver, C. M., Choveau, F. S., \& Shapiro, M. S. (2016). Clustering and Functional Coupling of Diverse Ion Channels and Signaling Proteins Revealed by Superresolution STORM Microscopy in Neurons. Neuron, 92(2), 461-478.

Zhang, Y., Chen, K., Sloan, S. A., Bennett, M. L., Scholze, A. R., O'Keeffe, S., . . Wu, J. Q. (2014). An RNA-sequencing transcriptome and splicing database of glia, neurons, and vascular cells of the cerebral cortex. J Neurosci, 34(36), 11929-11947.

Zhao, Z., Nelson, A. R., Betsholtz, C., \& Zlokovic, B. V. (2015). Establishment and Dysfunction of the Blood-Brain Barrier. Cell, 163(5), 1064-1078. 
Zhong, Z., Deane, R., Ali, Z., Parisi, M., Shapovalov, Y., O'Banion, M. K., . . Zlokovic, B. V. (2008). ALS-causing SOD1 mutants generate vascular changes prior to motor neuron degeneration. Nat Neurosci, 11(4), 420-422.

863

Zlokovic, B. V. (2008). The blood-brain barrier in health and chronic neurodegenerative

864 disorders. Neuron, 57(2), 178-201. doi:10.1016/j.neuron.2008.01.003

865 
Supplementary Figure S1 Total claudin-5 protein levels in bEND.3 cells and iBMECs TEER are elevated along days in culture

a

\begin{tabular}{lllll} 
& \multicolumn{4}{c}{ bEND3 } \\
\cline { 2 - 3 } $\begin{array}{l}\text { Pre- } \\
\text { confluence }\end{array}$ & day 0 & day 3 & day6 day 10 & day 13
\end{tabular}

anti-claudin-5

anti-GAPDH

b

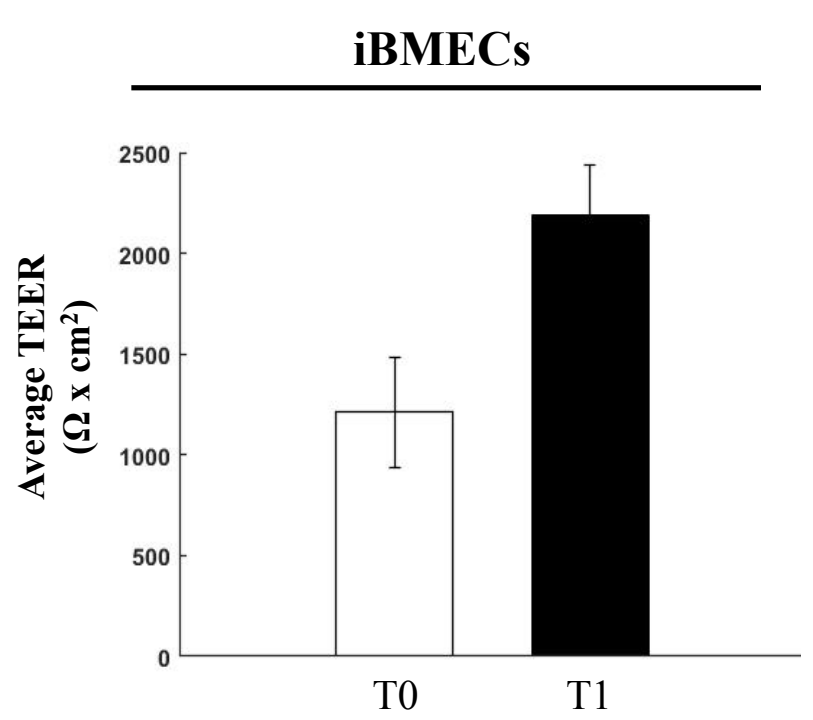


867 Supplementary Figures

868

869 Supplementary Figure S1: Total claudin-5 protein levels in bEND.3 cells and iBMECs

870 TEER are elevated with time in culture. a, Total claudin-5 protein levels in bEND.3 cells

871 rise with time in culture as shown in a representative western blot analysis. $n=4$. $\mathbf{b}$, Enhanced

872 TJ function demonstrated by doubling of TEER (average change in TEER across all

873 experiments) along 2-3 days of human brain microvascular endothelial-like cell (iBMECs)

874 culture ( $n=4$ experiments/12 inserts).

875 


\section{Supplementary Figure S2 Claudin-5 clustering properties}

a

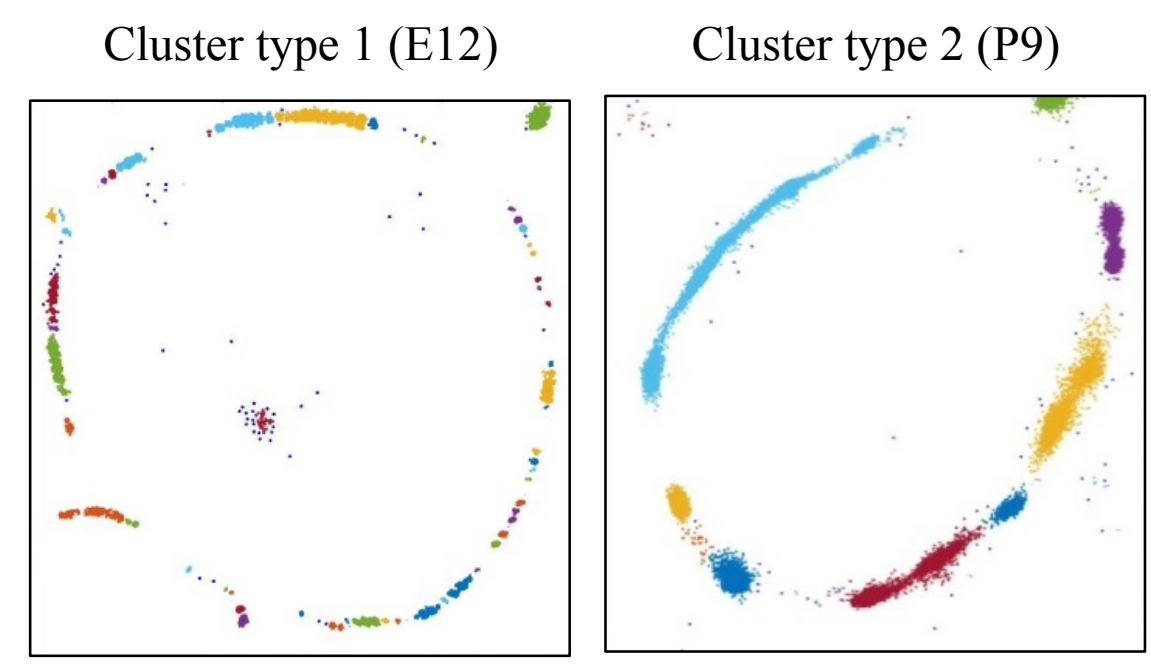

b

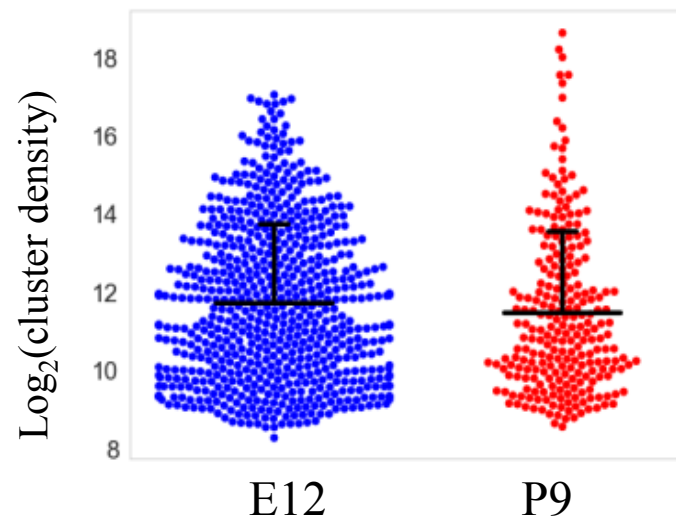


876 Supplementary Figure S2 Claudin-5 clustering properties. Analysis of Claudin-5

877 clustering properties along the developmental BBB maturation axis, using custom clustering

878 Matlab code (see methods for details). a, dSTORM imaging simulation of claudin-5

879 immunostaining of E12 and P9 cortical capillary cross-sections (example of the two types of

880 clustering simulations). We assumed that E12 capillaries had a more defused claudin-5

881 appearance with longer clusters, but further analysis revealed that these are composed of

882 many small clusters with relatively small gaps between them. Signals were defined to be

883 clustered if their 2D location was smaller than $70 \mathrm{~nm}$ threshold distance. Cluster pattern

884 visualization showing all points that belong to the same cluster with the same identifying

885 color. b, Claudin-5 clustering-properties analysis showed that there were about 2.6 times

886 more discrete clusters per capillary at E12 than at P9 (a set of 20 capillaries of each age).

887 There was no dramatic difference in the distribution of signal densities between the two

888 groups. $n=3$ pups/embryos, 20 capillaries, 657 clusters (E12) and 246 clusters (P9). Data are

889 mean \pm s.e.m. 
Supplementary Figure S3 Molecular organization of ZO1 and occludin in mouse cortical BBB TJs

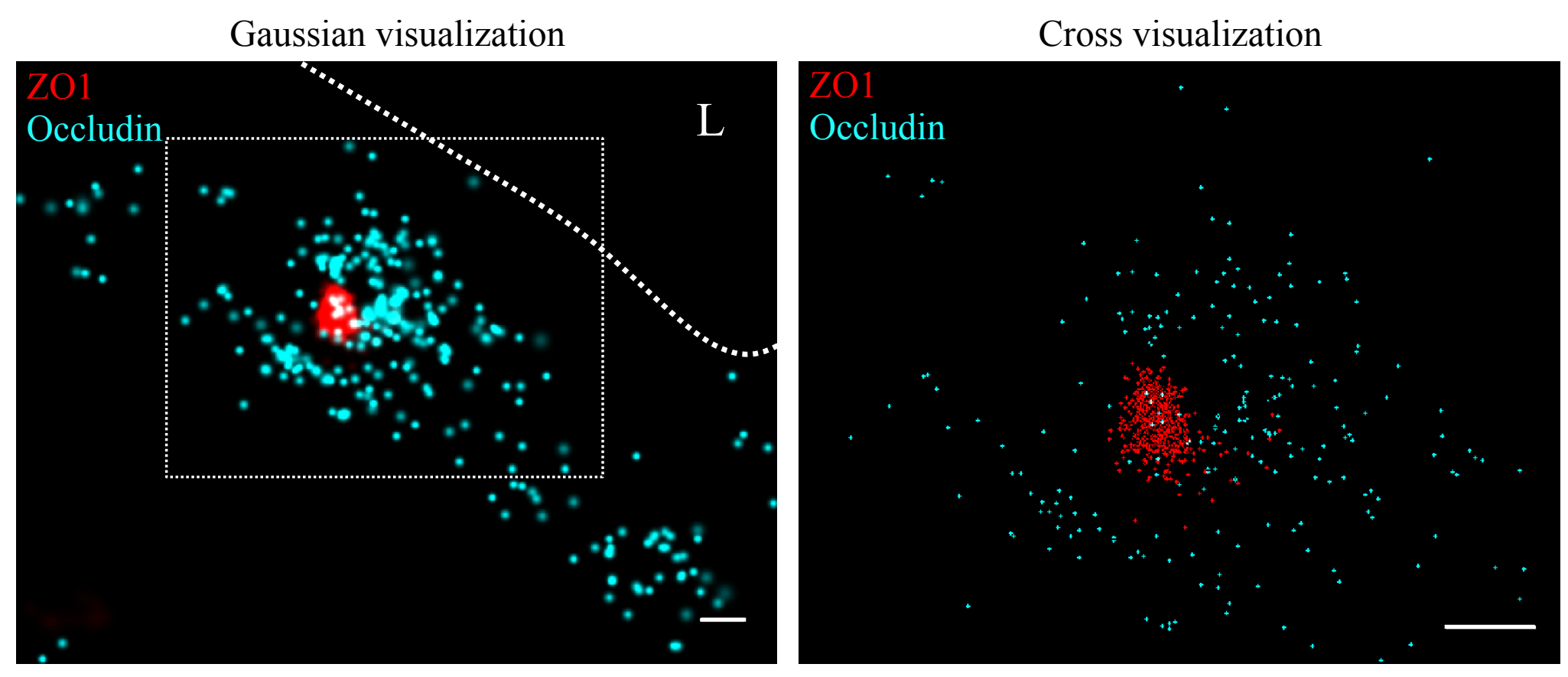


890 Supplementary Figure S3 Molecular organization of ZO1 and occludin in mouse cortical

891 BBB TJs. Nano-scale molecular organization of TJ proteins in cortical capillaries of wild-

892 type mice (E16). ZO1 display clustered organization (red) whereas occludin (cyan) was much

893 less organized in discrete clusters and had more dispersed organization patterns. 2D-STORM

894 imaging data (left) demonstrates that signals of TJ proteins are in close proximity and

895 sometimes overlap ('Gaussian visualization' in which signal intensity correlates with

896 localization precision). An inset with magnification (right) demonstrates the very high

897 molecular density of ZO1 and the more diffuse organization of occludin ('Cross visualization'

898 shows all resolved signals where each single-molecule signal displays as a cross). Scale bars,

899100 nm. $n=40$ capillaries of 4 wild-type pups. L - capillary lumen. 
Supplementary Figure S4 Claudin-5 clusters are in close proximity to ER and lysosomal markers

a
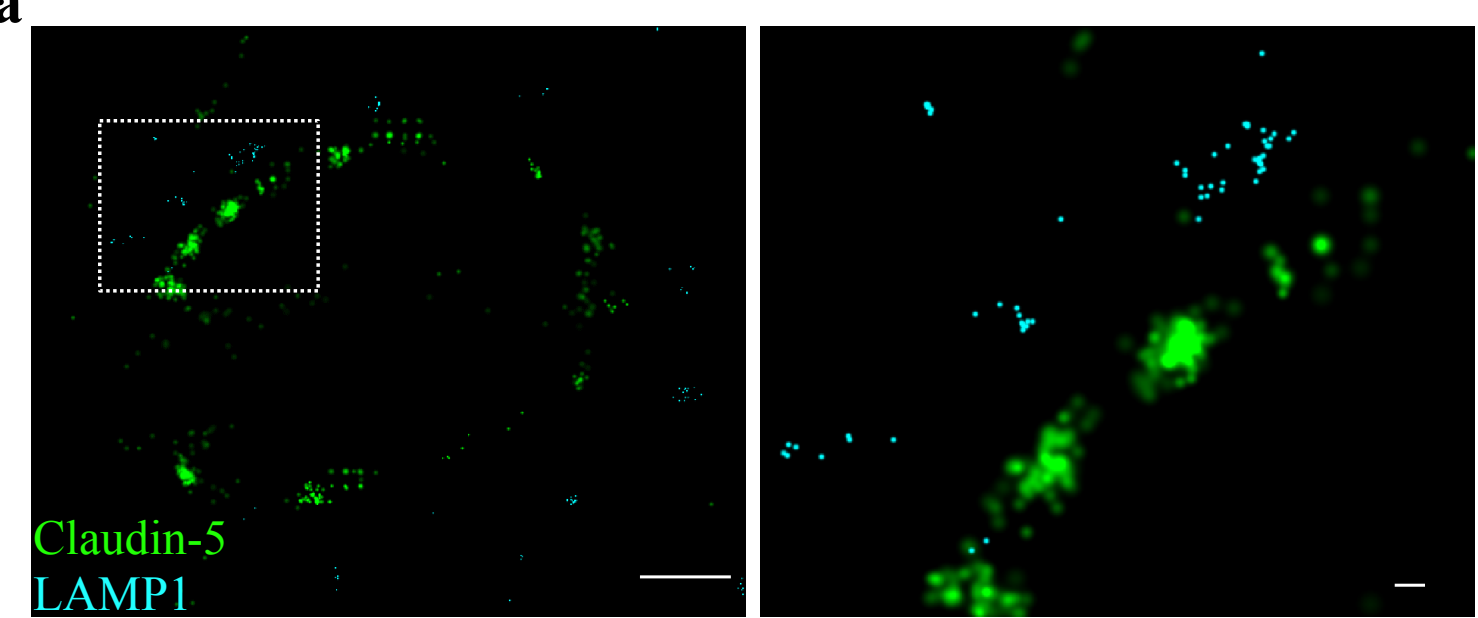

b
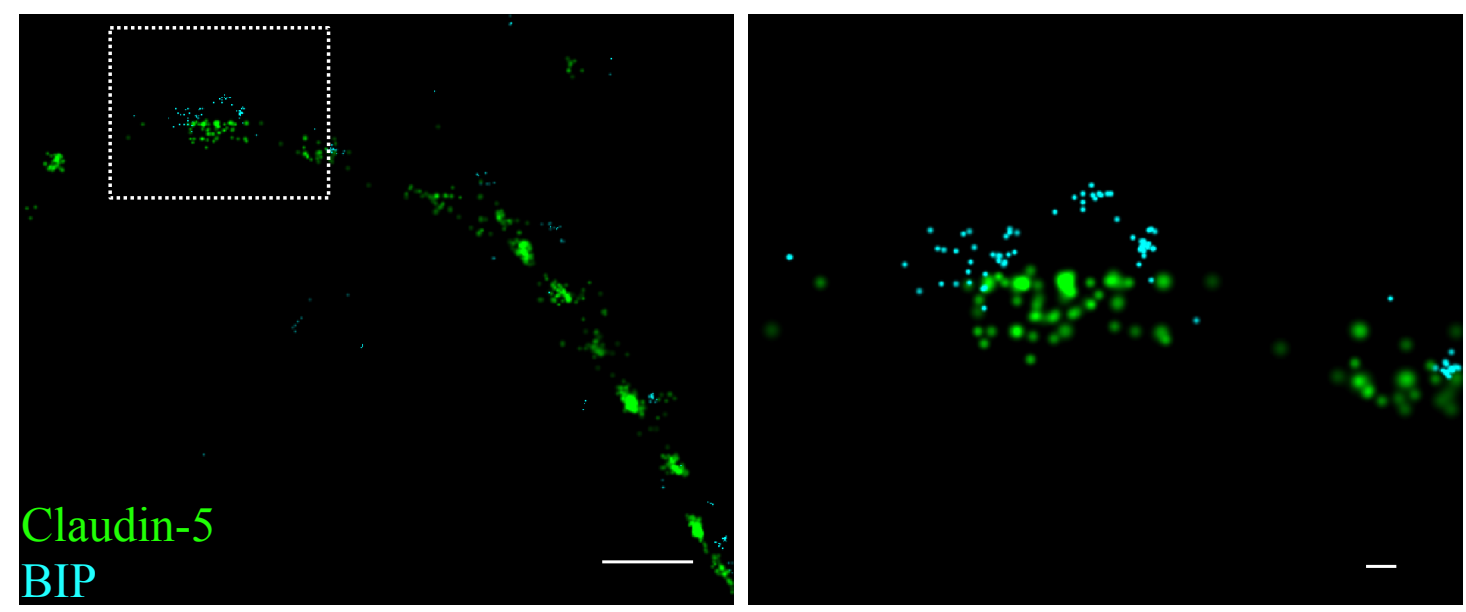


\section{Supplementary Figure S4: Claudin-5 clusters are in close proximity to ER and}

901 lysosomal markers. Capillary cross-sections presented multiple claudin-5 clusters, therefore

902 we tested potential claudin-5 localization to different cellular compartments. a, Claudin-5

903 clusters in close proximity with the lysosomal marker LAMP1, suggesting lysosome

904 localization. b, Claudin-5 clusters in close proximity with the ER marker BiP, suggesting ER

905 localization. Scale bars, $1 \mu \mathrm{m}$ and $0.1 \mu \mathrm{m}$ in insets. $\mathrm{n}=8$ capillaries.

906 
Supplementary Figure S5 clustering analysis - threshold distances

$\mathbf{a}$
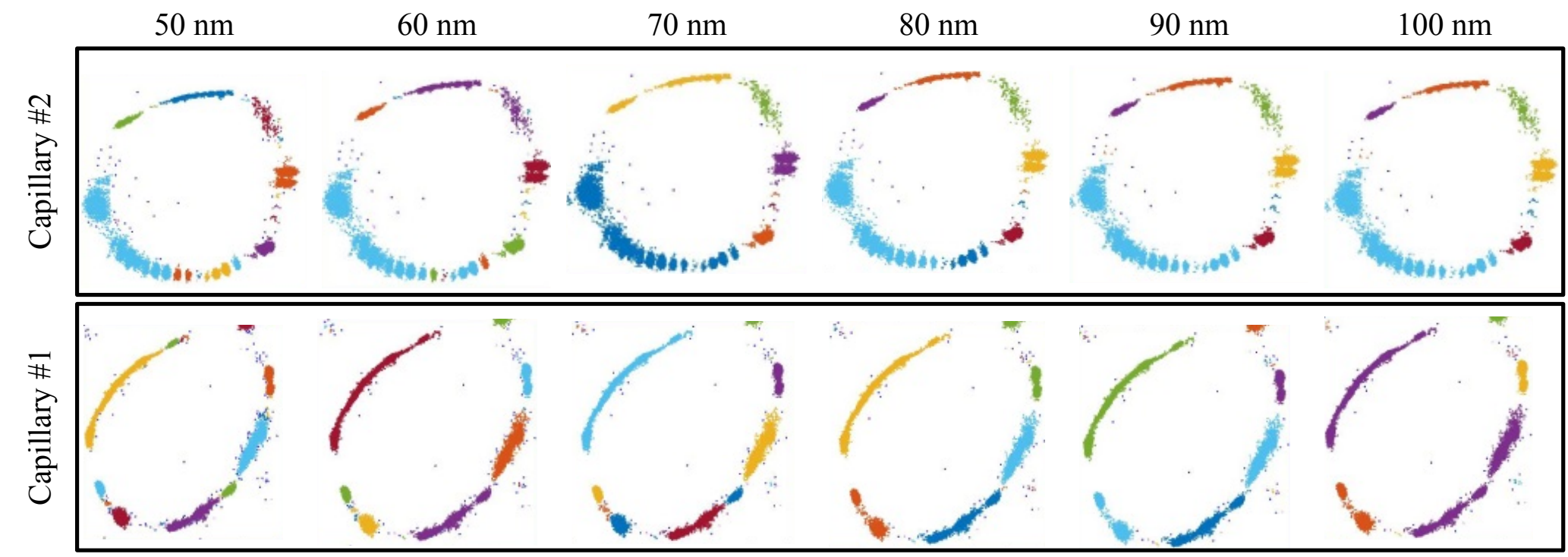

b

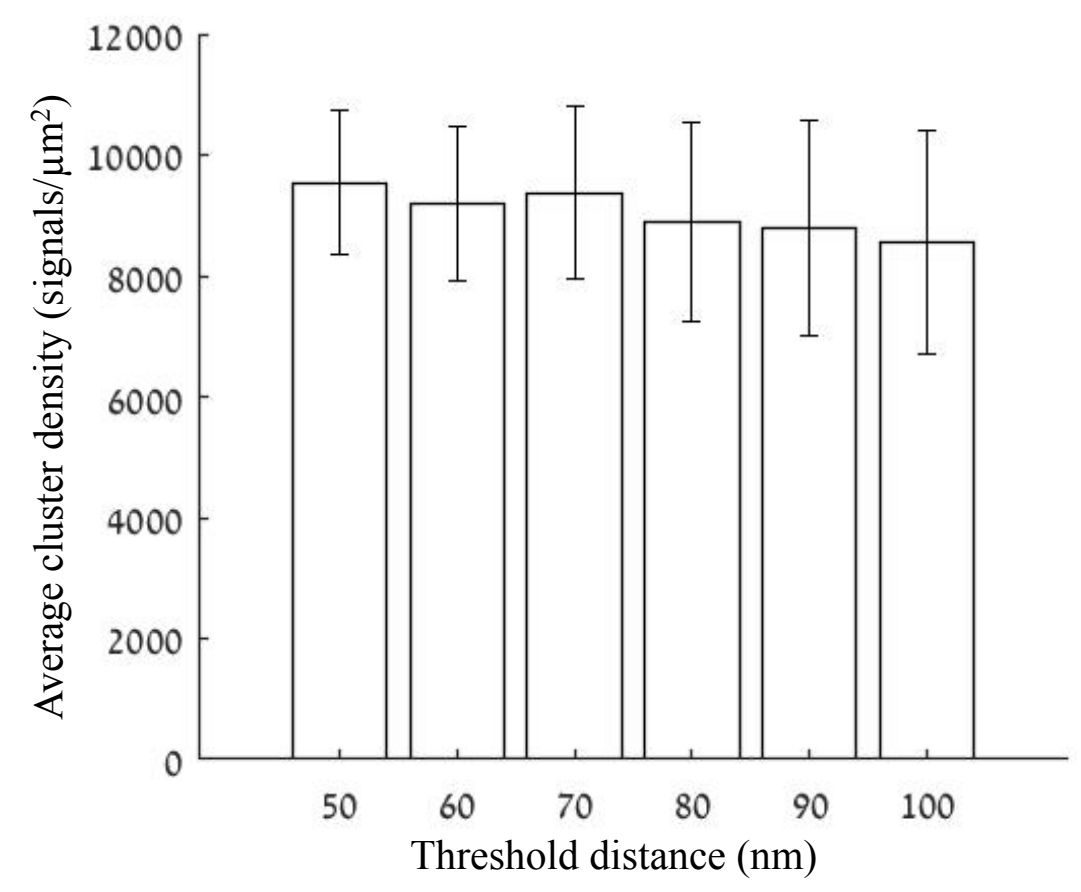


908 Supplementary Figure S5 Distance threshold used for quantifications of clustering

909 properties. Analysis of claudin-5 clustering properties was done using a custom clustering

910 Matlab code (see methods for details); our code calculated distances between each point and

911 all other points in the point pattern of a Single molecule localization microscopy (SMLM)

912 image. Then, we set a $70 \mathrm{~nm}$ distance threshold for defining molecules that belong to the

913 same cluster. This distance threshold used for quantifications was determined based on the

914 following parameters: minimal distance could not be below $40 \mathrm{~nm}$ (see methods for antibody

915 labeling strategy); BBB TJs covering continuous contact points, as we evaluated in published

916 TEM imaging data, range approximately up to $100 \mathrm{~nm}$. Finally, we preformed simulation of

917 claudin-5 density in clusters, measured in different threshold distances between 50-100 nm. a,

918 Two examples of simulations for claudin-5 cluster densities with different distance thresholds

919 in P9 capillaries. Point patterns are visualized, while showing all points that belong to the

920 same cluster with the same identifying color. Note that while clusters colors might change,

921 the overall cluster organization is similar across tested threshold distances. b, Claudin-5

922 cluster densities quantified using different distance thresholds did not yield significant

923 differences. $n=8$ capillaries. Data are mean \pm s.e.m.

924

925

926 